\title{
Article \\ Concurrent Heavy Metal Exposures and Idiopathic Dilated Cardiomyopathy: A Case-Control Study from the Katanga Mining Area of the Democratic Republic of Congo
}

\author{
Didier Malamba-Lez ${ }^{1,2, * \mathbb{D}}$, Désire Tshala-Katumbay ${ }^{3}$, Virginie Bito ${ }^{2}$, Jean-Michel Rigo ${ }^{2}(\mathbb{D}$, \\ Richie Kipenge Kyandabike ${ }^{1} \mathbb{D}$, Eric Ngoy Yolola ${ }^{1}$, Philippe Katchunga ${ }^{4}$, Béatrice Koba-Bora ${ }^{5}$ and \\ Dophra Ngoy-Nkulu ${ }^{1}$
}

1 Unit of Training and Research in Cardiology, Department of Internal Medicine, University of Lubumbashi, Lubumbashi 07610, Democratic Republic of the Congo; kipengerichie@gmail.com (R.K.K.); yololaericy@gmail.com (E.N.Y.); ngoynkuludophra@yahoo.fr (D.N.-N.)

2 Doctoral School for Medicine and Life Sciences, Hasselt University, 3590 Diepenbeek, Belgium; virginie.bito@uhasselt.be (V.B.); jeanmichel.rigo@uhasselt.be (J.-M.R.)

3 Department of Neurology and School of Public Health, Oregon Health \& Science University, Portland, OR 97007, USA; tshalad@ohsu.edu

4 Department of Internal Medicine, Official University of Bukavu, Bukavu 11102, Democratic Republic of the Congo; philkatch@yahoo.fr

check for updates

Citation: Malamba-Lez, D.; Tshala-Katumbay, D.; Bito, V.; Rigo, J.-M.; Kipenge Kyandabike, R.; Ngoy Yolola, E.; Katchunga, P.; Koba-Bora, B.; Ngoy-Nkulu, D. Concurrent Heavy Metal Exposures and Idiopathic Dilated Cardiomyopathy: A Case-Control Study from the Katanga Mining Area of the Democratic Republic of Congo. Int. J. Environ. Res. Public Health 2021, 18, 4956. https://doi.org/10.3390/ ijerph18094956

Academic Editor: Jaymie Meliker

Received: 20 March 2021

Accepted: 27 April 2021

Published: 6 May 2021

Publisher's Note: MDPI stays neutral with regard to jurisdictional claims in published maps and institutional affiliations.

Copyright: (c) 2021 by the authors. Licensee MDPI, Basel, Switzerland This article is an open access article distributed under the terms and conditions of the Creative Commons Attribution (CC BY) license (https:/ / creativecommons.org/licenses/by/ $4.0 /)$.
5 Department of Internal Medicine, Service of Neurology, University of Lubumbashi, Lubumbashi 07610, Democratic Republic of the Congo; kobora2001@yahoo.fr

* Correspondence: lezm@unilu.ac.cd; Tel.: +243-81-16-17-900

Abstract: Blood and/or urine levels of 27 heavy metals were determined by ICPMS in 41 patients with dilated cardiomyopathy (DCM) and 29 presumably healthy subjects from the Katanga Copperbelt (KC), in the Democratic Republic of Congo (DRC). After adjusting for age, gender, education level, and renal function, DCM probability was almost maximal for blood concentrations above 0.75 and $150 \mu \mathrm{g} / \mathrm{dL}$ for arsenic and copper, respectively. Urinary concentrations above 1 for chromium, 20 for copper, 600 for zinc, 30 for selenium, 2 for cadmium, 0.2 for antimony, 0.5 for thallium, and 0.05 for uranium, all in $\mu \mathrm{g} / \mathrm{g}$ of creatinine, were also associated with increased DCM probability. Concurrent and multiple exposures to heavy metals, well beyond permissible levels, are associated with increased probability for DCM. Study findings warrant screening for metal toxicity in case of DCM and prompt public health measures to reduce exposures in the KC, DRC.

Keywords: environmental exposures; heavy metals; idiopathic dilated cardiomyopathy; Katanga Copperbelt

\section{Introduction}

The Katanga Copperbelt (KC), with its significant copper and cobalt reserves, is a theater of intense mining and ore processing, producing more than half of DRC's and about $6 \%$ of the world's cobalt (the modern-day "oil" of a low-carbon economy) and copper, respectively [1-3]. Low-grade mining infrastructures compounding with artisanal mining, environmental degradation, and poor regulation account for concurrent and multiple environmental exposures to heavy metals and metalloids including but not limited to arsenic (As), cadmium $(\mathrm{Cd})$, cobalt $(\mathrm{Co})$, copper $(\mathrm{Cu})$, lead $(\mathrm{Pb})$, and zinc $(\mathrm{Zn})[4,5]$. Recent reports indicate that $\mathrm{KC}$ is one of the most polluted places in the world due to the contamination of water, soil, air, and food by toxic metals [6-17].

Previous studies in the KC have established an association between human exposure to toxic metals and disorders of neurodevelopment, decline in male and female fertility, and an increased occurrence of birth defects [18-22]. Other studies have shown that exposure to toxic metals confers a cardiovascular risk [23-25]. Toxic metals may have a direct effect on 
the functioning of cardiomyocyte membranes, ion channels, receptors, sarcomere, enzymes involved in the production of energy, and the antioxidant defense [26-35]. They may also lead to the dyshomeostasis of essential trace elements promoting adverse remodeling and ultimately dilated cardiomyopathy [36].

Recently in Lubumbashi, the largest mining city of the KC, severe cases of dilated cardiomyopathy burdened with high mortality were reported in relatively young patients, in contrast to findings in other populations. While risk factors such as hypertension, alcoholism, overweight and/or obesity, kidney dysfunction, atrial fibrillation, and difficult living conditions have been highlighted, the potential contribution of toxic metals to the high prevalence of idiopathic dilated cardiomyopathy (DCM) has been suspected previously [37]. Lubumbashi is growing in population with many challenges of urbanization and sanitation. There are many unpaved roads, dilapidated sewers dating from colonial times that are insufficient for draining of rainwater, and even effluents of mining companies that are located in the vicinity of homes. Garbage collection and recycling of dangerous products such as batteries and wrecks of vehicles is also a big challenge for the city. The majority of the population is poor, and artisanal mining is a major subsistence activity. Concurrent and multiple exposures to heavy metals, including some radioactive elements such as uranium, have been documented $[14,38]$. There is no universal health coverage, and the burden of major chronic diseases, including cardiac morbidities, lies on individuals $[3,16,39,40]$. In such contexts, identification of preventable causes of chronic illness and/or disability is of the utmost importance. In this study, we unveiled associations between idiopathic DCM and multiple exposures to heavy metals.

\section{Subjects and Methods}

\subsection{Subjects}

A total of 97 subjects seen prospectively for cardiovascular evaluations at the university clinic and the Lubumbashi's Centre of Cardiology were consecutively enrolled in the study between November 2017 through January 2019. They were at least 16 years old, male or female, living in the general population of the KC. Of these 97, $68(70.1 \%)$ had symptoms and signs of heart failure, elevated NT-proBNP, and DCM on echocardiography (either presumed idiopathic or postpartum cardiomyopathy). Twenty-nine subjects $(29.9 \%)$ had no cardiovascular disease after cardiovascular check-up and were therefore kept as controls in the present study. They all have a normal 12-lead ECG. Of the 68 above subjects, those over 70 years, with known diabetes; renal failure with the need for dialysis; positive HIV test; and transthoracic echocardiography supporting rheumatic or degenerative valvular heart disease, pericarditis, cor pulmonale, congenital defects, suspected ischemic heart disease, or dilation attributed to hypertension, were excluded from the study (Figure 1). 


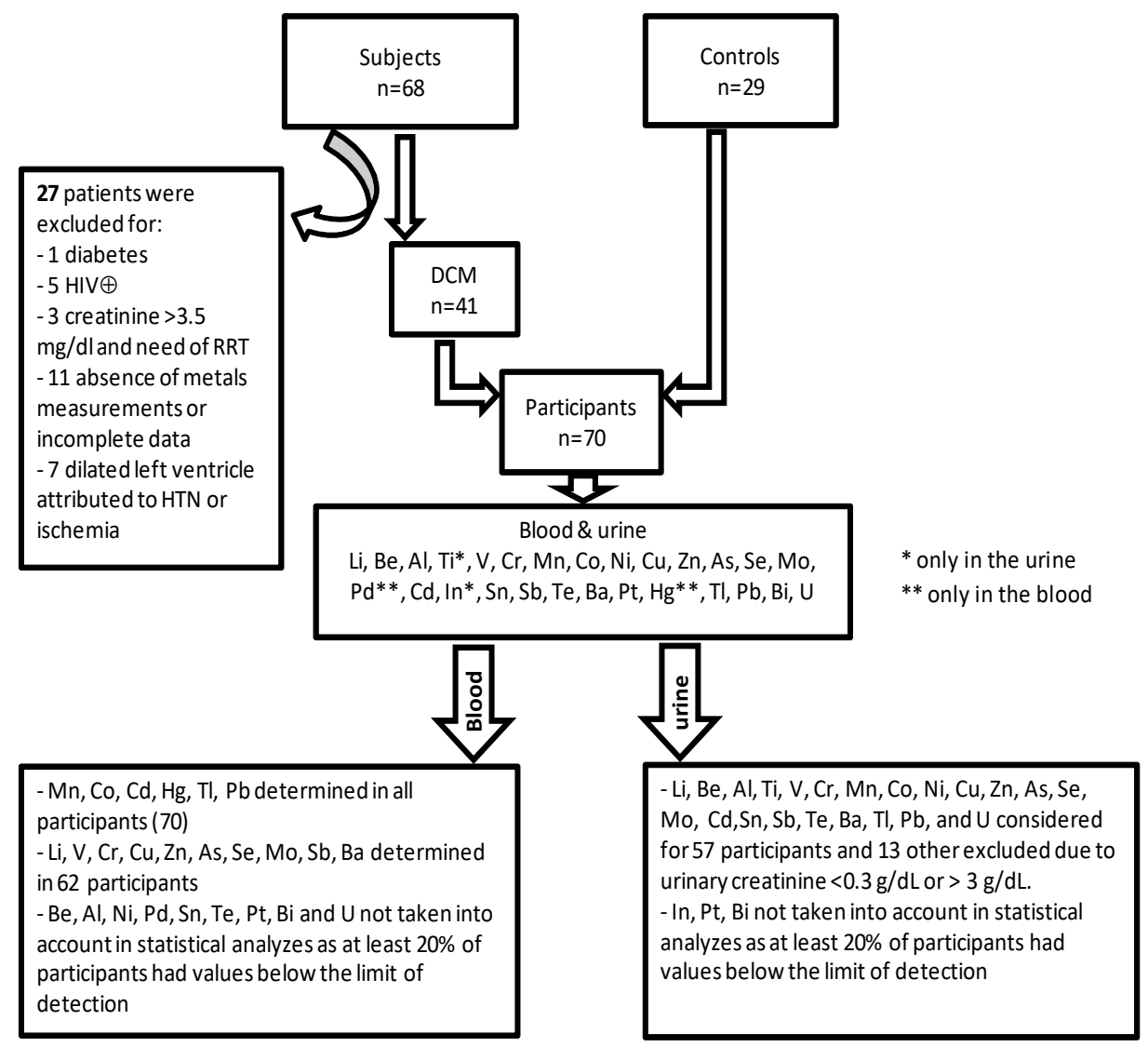

Figure 1. Patient selection, heavy metals measured in total blood and urine. LV denotes left ventricle, HIV $\oplus$ : human immunodeficiency virus positive test, RRT: renal replacement therapy, HTN: hypertension, DCM: dilated cardiomyopathy (idiopathic and postpartum cardiomyopathy).

\subsection{Readout Parameters and Measurements}

Demographic data, past medical history, signs, and symptoms of heart failure were collected using a standardized questionnaire.

Fasting routine blood (total blood cells count, INR, HIV test, sodium, potassium, magnesium, calcium, chloride, total cholesterol, HDL, LDL, triglycerides, urea, creatinine, uric acid, glycemia, AST, ALT, direct bilirubin, and indirect bilirubin) and urine tests (strip and microscopy) were performed in all subjects.

Transthoracic echocardiography was performed using a Vivid i ultraportable echo system (GE Medical Systems, Tirat Carmel, Israel). For each registration, five heartbeats were recorded. Cine loops were stored digitally and later analyzed in EchoPac version 113 software (GE Vingmed, Horten, Norway). Simpson's biplane method was applied for the assessment of left ventricle volumes and ejection fraction. 2D parameters and conventional Doppler parameters were measured according to recommendations [41,42].

Electrocardiogram was obtained with Cardiax PC system (Imed, Budapest, Hungary) and interpreted according to The Minnesota code manual of electrocardiography [43] and a 24-h Holter-ECG was recorded by a DMS 300-4A Holter recorder (DM Software, Hunfelden-Dauborn, Germany). Data from each Holter assessment were processed using CardioScan software (Cardioscan GmbH, Hamburg, Germany).

A spot sample of urine, which was voided directly into $40 \mathrm{~mL}$ polystyrene vials with screw caps, was obtained from each patient. A blood sample was drawn by a trained nurse from a brachial vein into a 4-mL BD Vacutainer tube with spray-coated K2EDTA. Blood and urine samples were obtained the day of the first visit or the morning of the following day. Levels of 27 heavy metals were measured: lithium ( $\mathrm{Li})$, beryllium (Be), aluminum ( $\mathrm{Al})$, titanium $(\mathrm{Ti})$, vanadium $(\mathrm{V})$, chromium $(\mathrm{Cr})$, manganese $(\mathrm{Mn})$, cobalt $(\mathrm{Co})$, nickel $(\mathrm{Ni})$, 
copper (Co), zinc $(\mathrm{Zn})$, arsenic (As), selenium (Se), molybdenum (Mo), palladium (Pd), cadmium $(\mathrm{Cd})$, indium $(\mathrm{In})$, tin $(\mathrm{Sn})$, antimony $(\mathrm{Sb})$, tellurium $(\mathrm{Te})$, barium $(\mathrm{Ba})$, platinum $(\mathrm{Pt})$, mercury $(\mathrm{Hg})$, thallium $(\mathrm{Tl})$, lead $(\mathrm{Pb})$, bismuth $(\mathrm{Bi})$, and uranium $(\mathrm{U})$. Palladium and mercury were only measured in the blood, while titanium and indium were only measured in the urine. Urinary concentrations of metals were reported in $\mu \mathrm{g} / \mathrm{g}$ of urinary creatinine. A value of half the urinary limit of detection (LOD) of beryllium, vanadium, manganese, and antimony was attributed to respectively 2, 7, 9, and 1 participants whose concentration was below the LOD. All measurements were carried out at the Louvain Centre for Toxicology and Applied Pharmacology (Université Catholique de Louvain, Belgium), using Agilent 7500 ce instrument (for urine) and Agilent $7500 \mathrm{cx}$ instrument (for blood). The same techniques already widely described were used [14,38,44]. Samples were anonymized, and analyses were performed blind.

\subsection{Statistical Analysis}

Shapiro-Wilk normality test was used to determine the distributions of metals levels, which were right-skewed, therefore summary results are presented as geometric means with their 95\% CIs. The $\mathrm{Zn} / \mathrm{Cu}$ ratio was calculated and included in the statistical analysis as previously of interest in other studies $[45,46]$. For the association studies, generalized linear models were used to obtain adjusted estimates, 95\% confidence interval, and $p$-value. Graphs of the fitted models showing DCM probability were produced using genmod procedure with SAS 9.4 TS Level1 M5 2016 (SAS Institute Inc., Cary, NC, USA). The level of statistical significance was set at $p<0.05$ (two-sided).

\section{Results}

\subsection{General Characteristics of Study Participants}

Table S1 (Supplementary Material) gives the general characteristics of patients with DCM. On average, participants were $48 \pm 14$ years old. Three-quarters (78\%) of them were hospitalized for an average of 14(8) days. As comorbidities and risk factors, 39\% of them regularly consumed clay and $27 \%$ misused alcohol at the time of diagnosis. They were symptomatic with fatigue $(93 \%)$, breathlessness $(83 \%)$, orthopnea $(83 \%)$, and early satiety $(85 \%)$. Several of them were congested with pulmonary rales $(49 \%)$, hepatomegaly $(63 \%)$, sacral $(66 \%)$, and legs edema $(71 \%)$. They were functioning in NYHA classes III and IV in $71 \%$ with a severely reduced ejection fraction $(21 \pm 8 \%)$. The initial treatment was essentially made of furosemide (95\%) and ACE-i (85\%).

Table S2 (Supplementary Material) compares patients with controls according to demography, anthropometry, education level, and routine biology. On average, patients were less educated, had elevated glycemia (108 vs. $88 \mathrm{mg} / \mathrm{dL}$ ), uric acid (11 vs. $6 \mathrm{mg} / \mathrm{dL}$ ), and C-Reactive protein ( $45 \mathrm{vs.} 7 \mathrm{mg} / \mathrm{L}$ ). They also had more renal dysfunction ( $41 \%$ vs. $7 \%$ ), electrolytes imbalance (sodium, potassium, and calcium), hepatic dysfunction (elevated ALT, bilirubin, and INR) and more hematologic disorders (WBC, red cell indices).

\subsection{Blood Concentrations of Heavy Metals and Dilated Cardiomyopathy}

Geometric means of cobalt, copper, and arsenic were significantly higher in patients than in controls, and beyond the reference values. Conversely, vanadium, zinc, antimony, and barium were significantly higher in controls than in patients, and also beyond the reference values. Higher $\mathrm{Zn} / \mathrm{Cu}$ ratio was found in controls (Table 1). 
Table 1. Concentrations ( $\mu \mathrm{g} / \mathrm{dL})$ of blood heavy metals in patients compared to controls.

\begin{tabular}{|c|c|c|c|c|}
\hline $\begin{array}{c}\text { Metal } \\
\text { (Reference Value) }\end{array}$ & $\begin{array}{c}\text { Controls }(n=29) \\
\text { GM }(95 \% \text { CL) } \\
\text { Mean } \pm \text { SD } \\
\text { Median (IQR) }\end{array}$ & $\begin{array}{c}\text { DCM }(n=41) \varnothing \\
\text { GM }(95 \% \text { CL) } \\
\text { Mean } \pm \text { SD } \\
\text { Median (IQR) }\end{array}$ & $\begin{array}{l}\text { GM Ratio } \\
(95 \% \text { CL) }\end{array}$ & $p$ \\
\hline $\begin{array}{c}\mathrm{Mn} \\
0.77 ¥\end{array}$ & $\begin{array}{c}1.59(1.41-1.81) \\
1.69 \pm 0.61 \\
1.59(0.58)\end{array}$ & $\begin{array}{c}1.56(1.39-1.76) \\
1.67 \pm 0.69 \\
1.64(0.80)\end{array}$ & $\begin{array}{c}1.02 \\
(0.86-1.22)\end{array}$ & 0.8094 \\
\hline $\begin{array}{c}\text { Co } \\
0.03 ¥\end{array}$ & $\begin{array}{c}0.06(0.04-0.08) \\
0.09 \pm 0.08 \\
0.05(0.07)\end{array}$ & $\begin{array}{c}0.12(0.08-0.16) \\
0.22 \pm 0.31 \\
0.09(0.14)\end{array}$ & $\begin{array}{c}0.50 \\
(0.31-0.82)\end{array}$ & 0.0063 \\
\hline $\begin{array}{c}\mathrm{Cd} \\
0.04 ¥\end{array}$ & $\begin{array}{c}0.16(0.15-0.17) \\
0.17 \pm 0.04 \\
0.16(0.04)\end{array}$ & $\begin{array}{c}0.13(0.08-0.21) \\
0.21 \pm 0.28 \\
0.14(0.09)\end{array}$ & $\begin{array}{c}1.24 \\
(0.86-1.31)\end{array}$ & 0.3636 \\
\hline $\mathrm{Hg}$ & $\begin{array}{c}0.26(0.22-0.31) \\
0.29 \pm 0.15 \\
0.27(0.13)\end{array}$ & $\begin{array}{c}0.26(0.22-0.31) \\
0.31 \pm 0.24 \\
0.26(0.13)\end{array}$ & $\begin{array}{c}0.99 \\
(0.77-1.28)\end{array}$ & 0.9663 \\
\hline $\begin{array}{c}\mathrm{Tl} \\
0.002 ¥\end{array}$ & $\begin{array}{c}0.0027(0.0024-0.0031) \\
0.0029 \pm 0.0012 \\
0.0027(0.0008)\end{array}$ & $\begin{array}{c}0.0028(0.002-0.0039) \\
0.0050 \pm 0.0082 \\
0.0036(0.0027)\end{array}$ & $\begin{array}{c}0.96 \\
(0.67-1.38)\end{array}$ & 0.8273 \\
\hline $\begin{array}{c}\mathrm{Pb} \\
1.88 ¥\end{array}$ & $\begin{array}{c}5.65(4.83-6.61) \\
6.13 \pm 2.59 \\
5.83(2.81)\end{array}$ & $\begin{array}{c}6.99(5.94-8.22) \\
8.02 \pm 4.94 \\
6.76(2.93)\end{array}$ & $\begin{array}{c}0.81 \\
(0.64-1.02)\end{array}$ & 0.0686 \\
\hline $\mathrm{Li}$ & $\begin{array}{c}0.21(0.08-0.61) \\
1.11 \pm 1.05 \\
1.22(1.89)\end{array}$ & $\begin{array}{c}0.10(0.07-0.16) \\
0.26 \pm 0.54 \\
0.10(0.11)\end{array}$ & $\begin{array}{c}2.08 \\
(0.67-6.45)\end{array}$ & 0.1981 \\
\hline $\begin{array}{c}\mathrm{V} \\
0.005 *\end{array}$ & $\begin{array}{c}0.02(0.01-0.05) \\
0.07 \pm 0.07 \\
0.52(0.13)\end{array}$ & $\begin{array}{c}0.006(0.004-0.01) \\
0.02 \pm 0.31 \\
0.007(0.008)\end{array}$ & $\begin{array}{c}3.5 \\
(1.40-8.98)\end{array}$ & 0.0086 \\
\hline $\begin{array}{c}\mathrm{Cr} \\
0.04\end{array}$ & $\begin{array}{c}0.20(0.18-0.22) \\
0.21 \pm 0.06 \\
0.19(0.04)\end{array}$ & $\begin{array}{c}0.18(0.15-0.21) \\
0.20 \pm 0.13 \\
0.17(0.07)\end{array}$ & $\begin{array}{c}1.12 \\
(0.94-1.33)\end{array}$ & 0.2136 \\
\hline $\begin{array}{c}\mathrm{Cu} \\
70-140 \neq\end{array}$ & $\begin{array}{c}110.6(105-116.4) \\
111.51 \pm 14.83 \\
109.71(20.50) \\
\end{array}$ & $\begin{array}{c}143.7(135.1-153) \\
145.88 \pm 25.74 \\
146.84(31.15)\end{array}$ & $\begin{array}{c}0.77 \\
(0.71-0.83)\end{array}$ & $<0.0001$ \\
\hline $\begin{array}{c}\mathrm{Zn} \\
580.5 ¥\end{array}$ & $\begin{array}{c}1196.9(992.1-1444) \\
1331.38 \pm 577.13 \\
1509.84(906.91)\end{array}$ & $\begin{array}{c}823.4(740.3-915.8) \\
864.21 \pm 307.60 \\
797.38(230.11)\end{array}$ & $\begin{array}{c}1.5 \\
(1.2-1.8)\end{array}$ & 0.0009 \\
\hline $\mathrm{Zn} / \mathrm{Cu}$ & $\begin{array}{c}10.8(8.8-13.2) \\
12.18 \pm 5.44 \\
13.33(8.02)\end{array}$ & $\begin{array}{c}5.7(5.1-6.4) \\
6.08 \pm 2.47 \\
5.39(1.95)\end{array}$ & $\begin{array}{c}1.9 \\
(1.5-2.4)\end{array}$ & $<0.0001$ \\
\hline $\begin{array}{c}\text { As } \\
0.17 ¥\end{array}$ & $\begin{array}{c}0.23(0.20-0.26) \\
0.25 \pm 0.09 \\
0.22(0.09)\end{array}$ & $\begin{array}{c}0.39(0.32-0.49) \\
0.47 \pm 0.33 \\
0.38(0.23)\end{array}$ & $\begin{array}{c}0.58 \\
(0.46-0.74)\end{array}$ & $<0.0001$ \\
\hline $\begin{array}{c}\text { Se } \\
12.5^{* *}\end{array}$ & $\begin{array}{c}12.6(11.8-13.4) \\
12.78 \pm 2.12 \\
13.12(3.34)\end{array}$ & $\begin{array}{c}11.9(11.1-12.8) \\
12.20 \pm 2.51 \\
11.82(3.28)\end{array}$ & $\begin{array}{c}1.05 \\
(0.9-1.2)\end{array}$ & 0.2820 \\
\hline $\begin{array}{c}\text { Mo } \\
0.1-0.3 \ddagger\end{array}$ & $\begin{array}{c}0.18(0.16-0.21) \\
0.20 \pm 0.11 \\
0.17(0.07)\end{array}$ & $\begin{array}{c}0.21(0.18-0.25) \\
0.25 \pm 0.23 \\
0.19(0.08)\end{array}$ & $\begin{array}{c}0.87 \\
(0.69-1.09)\end{array}$ & 0.2385 \\
\hline $\begin{array}{c}\mathrm{Sb} \\
0.005\end{array}$ & $\begin{array}{c}1.11(0.96-1.29) \\
1.19 \pm 0.46 \\
1.18(0.47)\end{array}$ & $\begin{array}{c}0.56(0.49-0.64) \\
0.61 \pm 0.29 \\
0.57(0.27)\end{array}$ & $\begin{array}{c}1.98 \\
(1.63-2.42)\end{array}$ & $<0.0001$ \\
\hline $\begin{array}{c}\mathrm{Ba} \\
0.05-0.25\end{array}$ & $\begin{array}{c}0.31(0.2-0.48) \\
0.53 \pm 0.53 \\
0.33(0.68)\end{array}$ & $\begin{array}{c}0.13(0.09-0.16) \\
0.18 \pm 0.29 \\
0.11(0.05)\end{array}$ & $\begin{array}{c}2.45 \\
(1.50-4.01)\end{array}$ & 0.0006 \\
\hline
\end{tabular}

Ø: Lithium, vanadium, chromium, copper, zinc, arsenic, selenium, molybdenum, antimony, and barium were measured in only 33 patients with DCM. ‡: WHO. Trace elements in human nutrition and health. 1996. ¥: Nisse C et al., Blood and urinary levels of metals and metalloids in the general adult population of Northern France: The IMEPOGE study, 2008-2010. Int J Hyg Environ Health. 2017;220(2 Pt B):341-63. *: Agency for Toxic Substances and Disease Registry (ATSDR). 2012. Toxicological Profile for Vanadium. Atlanta, GA: U.S. Department of Health and Human Services, Public Health Services. **: Agency for Toxic Substances and Disease Registry (ATSDR). 2003. Toxicological Profile for Selenium (Update). Atlanta, GA: U.S. Department of Health and Human Services, Public Health Service. 
DCM prediction models with adjustment for age, sex, education, and renal function (Table 2) revealed that higher blood levels of arsenic and copper were independently associated with DCM. However, a high $\mathrm{Zn} / \mathrm{Cu}$ ratio, and high concentrations of vanadium, zinc, antimony, and barium, were not associated with DCM.

Table 2. Adjusted models of DCM as a function of blood metal levels.

\begin{tabular}{|c|c|c|c|c|c|c|}
\hline Variable & Coefficient & SE & \multicolumn{2}{|c|}{ Wald $95 \%$ Confidence Limits } & Wald $X^{2}$ & $p$-Value \\
\hline \multicolumn{7}{|c|}{ Arsenic Model } \\
\hline Intercept & -2.84 & 1.65 & -6.06 & 0.39 & 2.97 & 0.0850 \\
\hline As & 9.04 & 3.44 & 2.28 & 15.79 & 6.88 & 0.0087 \\
\hline Age & -0.001 & 0.03 & -0.06 & 0.06 & 0.00 & 0.9639 \\
\hline Male sex & 0.48 & 0.70 & -0.89 & 1.85 & 0.47 & 0.4909 \\
\hline Education $\mathrm{X}$ & -1.14 & 0.74 & -2.59 & 0.29 & 2.43 & 0.1192 \\
\hline GFR $<60$ & 2.21 & 1.00 & 0.24 & 4.17 & 4.83 & 0.0279 \\
\hline \multicolumn{7}{|c|}{ Copper model } \\
\hline Intercept & -13.49 & 3.98 & -21.29 & -5.68 & 11.48 & 0.0007 \\
\hline $\mathrm{Cu}$ & 0.09 & 0.02 & 0.04 & 0.14 & 13.07 & 0.0003 \\
\hline Age & 0.02 & 0.03 & -0.04 & 0.09 & 0.50 & 0.4776 \\
\hline Male sex & 1.46 & 0.87 & -0.24 & 3.15 & 2.85 & 0.0915 \\
\hline Education & -0.79 & 0.80 & -2.37 & 0.77 & 0.99 & 0.3195 \\
\hline GFR $<60$ & 1.18 & 1.15 & -1.07 & 3.44 & 1.06 & 0.3042 \\
\hline \multicolumn{7}{|c|}{ Vanadium model } \\
\hline Intercept & 1.39 & 1.49 & -1.53 & 4.31 & 0.87 & 0.3517 \\
\hline Vanadium & -23.91 & 8.93 & -41.42 & -6.40 & 7.16 & 0.0074 \\
\hline Age & -0.01 & 0.03 & -0.08 & 0.05 & 0.22 & 0.6394 \\
\hline Male sex & 0.30 & 0.68 & -1.04 & 1.65 & 0.19 & 0.6589 \\
\hline Education & -1.18 & 0.70 & -2.55 & 0.19 & 2.83 & 0.0925 \\
\hline GFR $<60$ & 2.75 & 1.13 & 0.54 & 4.96 & 5.95 & 0.0147 \\
\hline \multicolumn{7}{|c|}{ Zinc model } \\
\hline Intercept & 3.36 & 1.86 & -0.28 & 7.00 & 3.28 & 0.0701 \\
\hline Zinc & -0.003 & 0.0009 & -0.005 & -0.001 & 9.53 & 0.0020 \\
\hline Age & -0.02 & 0.03 & -0.08 & 0.05 & 0.21 & 0.6437 \\
\hline Male sex & 0.79 & 0.73 & -0.63 & 2.22 & 1.18 & 0.2769 \\
\hline Education & -1.39 & 0.74 & -2.84 & 0.06 & 3.54 & 0.0598 \\
\hline GFR $<60$ & 3.32 & 1.31 & 0.75 & 5.88 & 6.43 & 0.0112 \\
\hline \multicolumn{7}{|c|}{ Antimony model } \\
\hline Intercept & 4.89 & 2.16 & 0.66 & 9.13 & 5.12 & 0.0236 \\
\hline Antimony & -4.65 & 1.25 & -7.09 & -2.20 & 13.87 & 0.0002 \\
\hline Age & -0.02 & 0.04 & -0.09 & 0.05 & 0.40 & 0.5284 \\
\hline Male sex & 0.58 & 0.82 & -1.01 & 2.19 & 0.52 & 0.4720 \\
\hline Education & -1.42 & 0.84 & -3.07 & 0.22 & 2.89 & 0.0892 \\
\hline GFR $<60$ & 3.58 & 1.66 & 0.33 & 6.83 & 4.65 & 0.0310 \\
\hline \multicolumn{7}{|c|}{ Barium model } \\
\hline Intercept & 1.32 & 1.40 & -1.43 & 4.06 & 0.89 & 0.3459 \\
\hline Barium & -2.73 & 1.24 & -5.17 & -0.29 & 4.81 & 0.0284 \\
\hline Age & -0.01 & 0.03 & -0.07 & 0.05 & 0.15 & 0.6997 \\
\hline Male sex & 0.19 & 0.65 & -1.09 & 1.48 & 0.09 & 0.7673 \\
\hline Education & -1.18 & 0.67 & -2.49 & 0.13 & 3.13 & 0.0769 \\
\hline GFR $<60$ & 2.54 & 1.01 & 0.57 & 4.51 & 6.36 & 0.0117 \\
\hline \multicolumn{7}{|c|}{ Ratio Zinc/Copper } \\
\hline Intercept & 3.63 & 2.03 & -0.35 & 7.61 & 3.20 & 0.0738 \\
\hline Zinc/copper & -0.46 & 0.14 & -0.74 & -0.19 & 11.01 & 0.0009 \\
\hline Age & -0.01 & 0.04 & -0.09 & 0.07 & 0.06 & 0.8053 \\
\hline Male sex & 1.44 & 0.89 & -0.31 & 3.20 & 2.59 & 0.1077 \\
\hline Education & -1.31 & 0.81 & -2.89 & 0.27 & 2.63 & 0.1050 \\
\hline GFR $<60$ & 3.73 & 1.71 & 0.38 & 7.07 & 4.76 & 0.0291 \\
\hline
\end{tabular}

$\mathrm{X}$ : Being highly educated (university or post-university) often correlates with higher socio-economic status in the KC settings. 
Probability curves of the likelihood of developing DCM as a function of heavy metals blood concentrations are shown in Figure 2. A sharp rise of DCM probability at levels of blood copper between 100 and $150 \mu \mathrm{g} / \mathrm{dL}$ was observed. Above $150 \mu \mathrm{g} / \mathrm{dL}$, the probability was almost $100 \%$. The same trend was seen with arsenic above $0.75 \mu \mathrm{g} / \mathrm{dL}$. In contrast, DCM probability decreases as blood concentrations of vanadium (C), zinc (D), antimony (E), and the $\mathrm{Zn} / \mathrm{Cu}$ ratio increase.
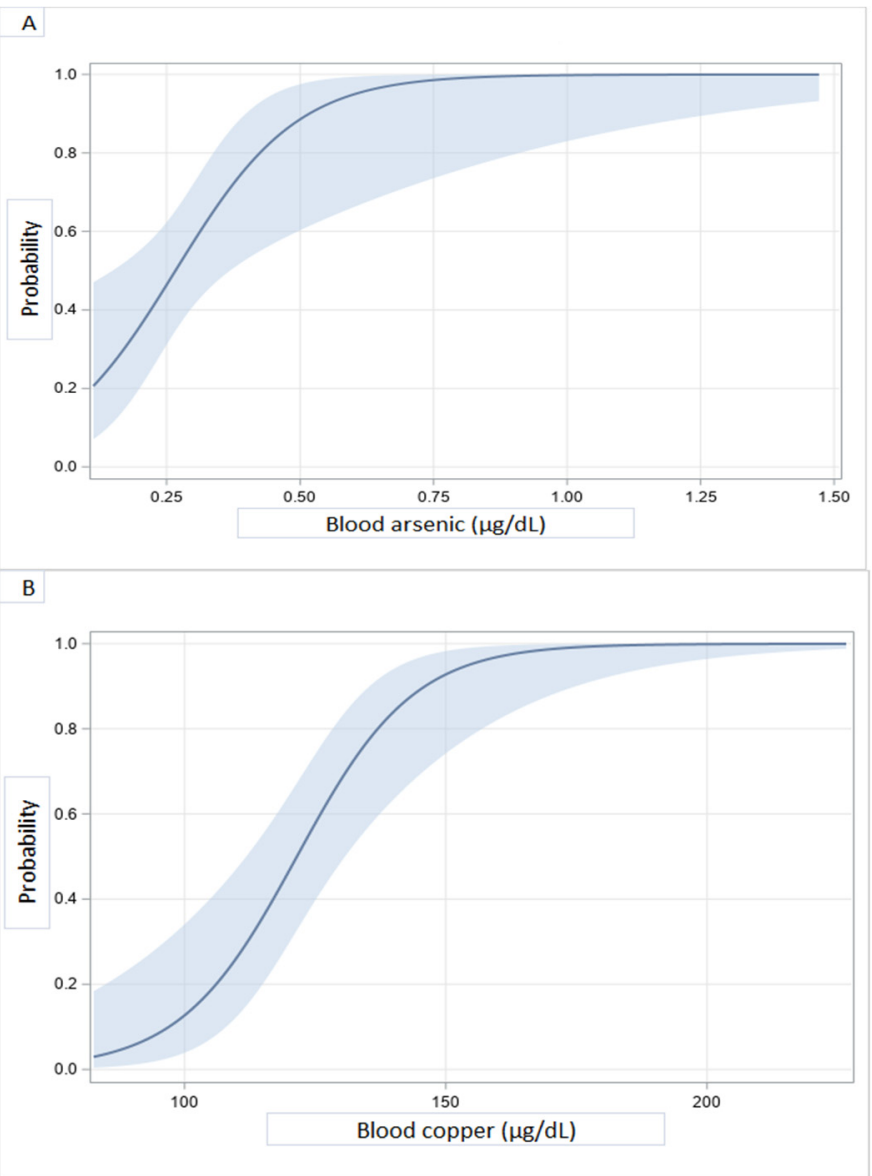

Figure 2. Cont. 

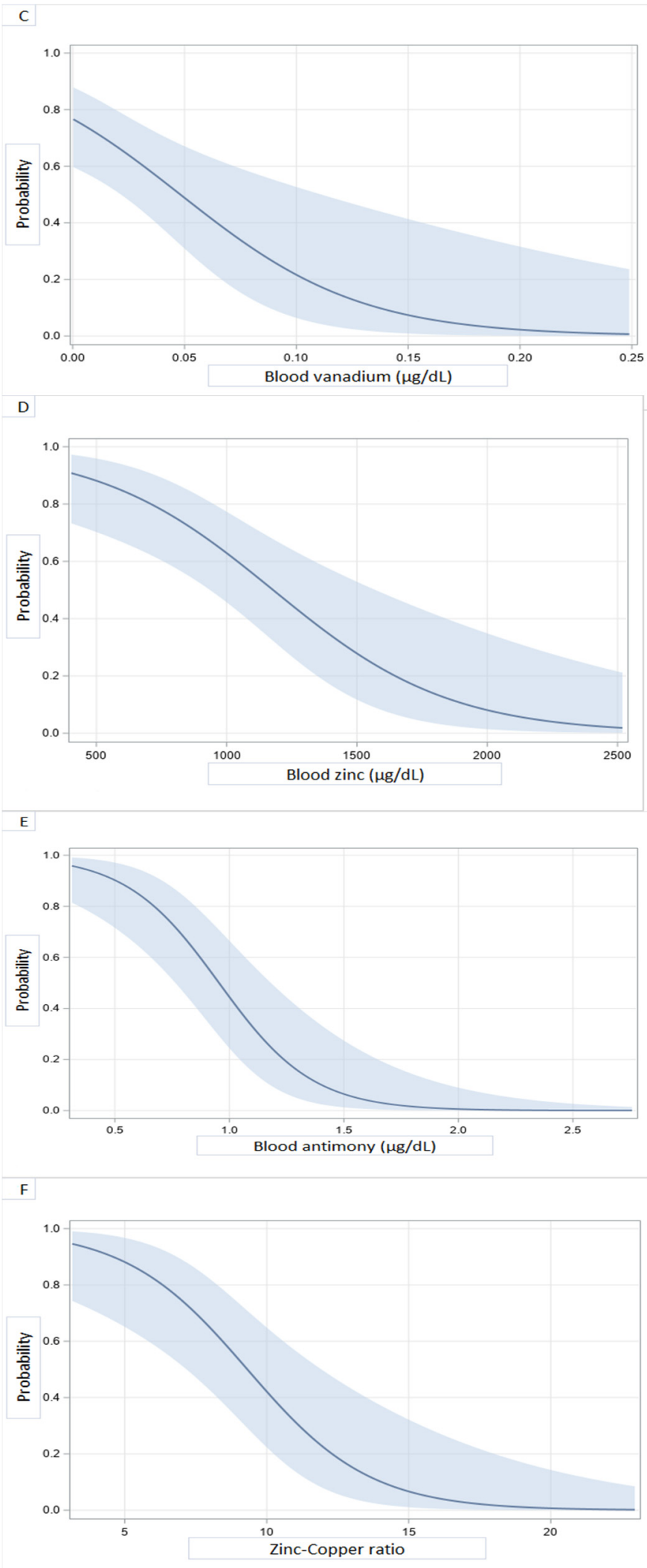

Figure 2. Illustrative predicted probabilities of DCM as a function of blood heavy metal concentrations: arsenic (A), copper (B), vanadium (C), zinc (D), antimony (E), and zinc/copper ratio (F). 


\subsection{Urine Concentrations of Heavy Metals and Dilated Cardiomyopathy}

As summarized in Table 3, the concentrations of beryllium, chromium, manganese, cobalt, copper, zinc, selenium, cadmium, tin, antimony, thallium, and uranium were well beyond the reference values and significantly higher in patients than in controls.

Table 3. Concentrations ( $\mu \mathrm{g} / \mathrm{g}$ creatinine) of metals in urine from DCM patients and controls.

\begin{tabular}{|c|c|c|c|c|}
\hline $\begin{array}{c}\text { Metal } \\
\text { (Reference Value) }\end{array}$ & $\begin{array}{l}\text { Controls }(n=25) \\
\text { GM }(95 \% \text { CL) } \\
\text { Mean } \pm \text { SD } \\
\text { Median (IQR) }\end{array}$ & $\begin{array}{c}\text { DCM }(n=32) \\
\text { GM }(95 \% \text { CL) } \\
\text { Mean } \pm \text { SD } \\
\text { Median (IQR) }\end{array}$ & $\begin{array}{l}\text { GM Ratio } \\
(95 \% \text { CL) }\end{array}$ & $p$ \\
\hline $\begin{array}{c}\mathrm{Li} \\
21.5\end{array}$ & $\begin{array}{c}13.93(11.98-16.19) \\
14.92 \pm 6.14 \\
13.44(6.04)\end{array}$ & $\begin{array}{c}16.06(12.55-20.57) \\
20.52 \pm 18.51 \\
15.25(13.44)\end{array}$ & $\begin{array}{c}0.87 \\
(0.65-1.15)\end{array}$ & 0.3189 \\
\hline $\mathrm{Be}$ & $\begin{array}{c}0.0009(0007-0.0012) \\
0.0011 \pm 0009 \\
0.0008(0004)\end{array}$ & $\begin{array}{c}0.0029(0.0015-0.0056) \\
0.041 \pm 0.15 \\
0.0022(0.0059)\end{array}$ & $\begin{array}{c}0.31 \\
(0.16-0.62)\end{array}$ & 0.0015 \\
\hline $\begin{array}{c}\mathrm{Al} \\
2.04\end{array}$ & $\begin{array}{c}10.62(7.97-14.15) \\
14.23 \pm 15.39 \\
9.59(6.42)\end{array}$ & $\begin{array}{c}15.62(10.22-23.88) \\
97.42 \pm 438.12 \\
11.25(10.62)\end{array}$ & $\begin{array}{c}0.68 \\
(0.41-1.12)\end{array}$ & 0.1292 \\
\hline $\mathrm{Ti}$ & $\begin{array}{c}30.87(25.19-37.82) \\
34.49 \pm 16.15 \\
29.40(25.48)\end{array}$ & $\begin{array}{c}31.13(23.07-42.02) \\
39.95 \pm 25.09 \\
34.43(29.65)\end{array}$ & $\begin{array}{c}0.99 \\
(0.69-1.41)\end{array}$ & 0.9612 \\
\hline $\begin{array}{c}\mathrm{V} \\
0.22\end{array}$ & $\begin{array}{c}1.46(0.73-2.93) \\
3.33 \pm 3.49 \\
2.07(3.24) \\
\end{array}$ & $\begin{array}{c}1.53(0.91-2.57) \\
2.95 \pm 3.08 \\
1.92(2.67)\end{array}$ & $\begin{array}{c}0.96 \\
(0.42-2.19)\end{array}$ & 0.9178 \\
\hline $\begin{array}{l}\mathrm{Cr} \\
0.11\end{array}$ & $\begin{array}{c}0.18(0.14-0.22) \\
0.20 \pm 0.13 \\
0.16(0.08)\end{array}$ & $\begin{array}{c}0.36(0.24-0.54) \\
1.00 \pm 2.91 \\
0.29(0.39)\end{array}$ & $\begin{array}{c}0.49 \\
(0.32-0.78)\end{array}$ & 0.0033 \\
\hline $\begin{array}{c}\mathrm{Mn} \\
<0.043\end{array}$ & $\begin{array}{c}0.12(0.06-0.21) \\
0.28 \pm 0.37 \\
0.11(0.27)\end{array}$ & $\begin{array}{c}0.39(0.19-0.81) \\
3.36 \pm 8.65 \\
0.29(1.05)\end{array}$ & $\begin{array}{c}0.29 \\
(0.11-0.78)\end{array}$ & 0.0145 \\
\hline $\begin{array}{l}\text { Co } \\
0.2\end{array}$ & $\begin{array}{c}1.18(0.73-1.92) \\
2.45 \pm 3.58 \\
0.97(1.84)\end{array}$ & $\begin{array}{l}2.75(1.98-3.82) \\
4.31 \pm 5.08 \\
2.24(3.46)\end{array}$ & $\begin{array}{c}0.43 \\
(0.25-0.75)\end{array}$ & 0.0034 \\
\hline $\begin{array}{c}\mathrm{Ni} \\
1.79\end{array}$ & $\begin{array}{c}1.19(0.94-1.51) \\
1.39 \pm 0.75 \\
1.18(1.24)\end{array}$ & $\begin{array}{c}1.49(1.16-1.92) \\
1.87 \pm 1.33 \\
1.57(1.44)\end{array}$ & $\begin{array}{c}0.79 \\
(0.57-1.13)\end{array}$ & 0.1967 \\
\hline $\begin{array}{l}\mathrm{Cu} \\
6.84\end{array}$ & $\begin{array}{c}7.38(6.45-8.43) \\
7.78 \pm 2.81 \\
7.06(2.48) \\
\end{array}$ & $\begin{array}{c}25.76(19.93-33.29) \\
32.48 \pm 23.22 \\
28.52(25.87)\end{array}$ & $\begin{array}{c}0.29 \\
(0.22-0.38)\end{array}$ & $<0.0001$ \\
\hline $\begin{array}{l}\mathrm{Zn} \\
246\end{array}$ & $\begin{array}{c}221.10(176.70-276.07) \\
250.38 \pm 116.76 \\
256.67(167.09)\end{array}$ & $\begin{array}{c}1033.80(818.5-1305.7) \\
1256.08 \pm 820.55 \\
1007.14(821.22)\end{array}$ & $\begin{array}{c}0.21 \\
(0.15-0.29)\end{array}$ & $<0.0001$ \\
\hline $\begin{array}{c}\text { As } \\
13.7\end{array}$ & $\begin{array}{c}20.36(14.99-27.65) \\
27.19 \pm 24.59 \\
19.97(20.48)\end{array}$ & $\begin{array}{c}27.06(20.41-35.89) \\
35.21 \pm 24.55 \\
29.06(29.68)\end{array}$ & $\begin{array}{c}0.75 \\
(0.49-1.13)\end{array}$ & 0.1689 \\
\hline $\begin{array}{l}\mathrm{Se} \\
21.6\end{array}$ & $\begin{array}{c}17.42(15.48-19.59) \\
18.09 \pm 5.07 \\
18.54(5.63)\end{array}$ & $\begin{array}{c}24.53(21.70-27.73) \\
25.98 \pm 9.23 \\
22.85(12.10)\end{array}$ & $\begin{array}{c}0.71 \\
(0.59-0.84)\end{array}$ & 0.0001 \\
\hline $\begin{array}{l}\text { Mo } \\
29.8\end{array}$ & $\begin{array}{c}60.46(41.95-87.13) \\
84.94 \pm 74.05 \\
65.18 \pm 38.00\end{array}$ & $\begin{array}{c}55.17(38.23-79.60) \\
92.35 \pm 137.29 \\
49.98(54.58)\end{array}$ & $\begin{array}{c}1.09 \\
(0.65-1.83)\end{array}$ & 0.4882 \\
\hline
\end{tabular}


Table 3. Cont.

\begin{tabular}{|c|c|c|c|c|}
\hline $\begin{array}{c}\text { Metal } \\
\text { (Reference Value) }\end{array}$ & $\begin{array}{c}\text { Controls }(n=25) \\
\text { GM }(95 \% \text { CL) } \\
\text { Mean } \pm \text { SD } \\
\text { Median (IQR) }\end{array}$ & $\begin{array}{c}\text { DCM }(n=32) \\
\text { GM }(95 \% \text { CL) } \\
\text { Mean } \pm \text { SD } \\
\text { Median (IQR) }\end{array}$ & $\begin{array}{l}\text { GM Ratio } \\
(95 \% \text { CL) }\end{array}$ & $p$ \\
\hline $\begin{array}{c}\mathrm{Cd} \\
0.22\end{array}$ & $\begin{array}{c}0.61(0.48-0.76) \\
0.72 \pm 0.54 \\
0.57(0.41)\end{array}$ & $\begin{array}{c}1.48(1.15-1.90) \\
1.94 \pm 1.96 \\
1.44(1.29)\end{array}$ & $\begin{array}{c}0.41 \\
(0.29-0.58)\end{array}$ & $<0.0001$ \\
\hline $\begin{array}{c}\text { Sn } \\
0.35\end{array}$ & $\begin{array}{c}0.23(0.19-0.29) \\
0.26 \pm 0.14 \\
0.21(0.11)\end{array}$ & $\begin{array}{c}3.59(1.79-7.19) \\
11.24 \pm 14.55 \\
6.86(16.66)\end{array}$ & $\begin{array}{c}0.07 \\
(0.03-0.13)\end{array}$ & $<0.0001$ \\
\hline $\begin{array}{c}\mathrm{Sb} \\
0.04\end{array}$ & $\begin{array}{c}0.05(0.04-0.06) \\
0.05 \pm 0.04 \\
0.046(0.019)\end{array}$ & $\begin{array}{c}0.08(0.05-0.11) \\
0.11 \pm 0.09 \\
0.08(0.14)\end{array}$ & $\begin{array}{c}0.64 \\
(0.42-0.97)\end{array}$ & 0.0351 \\
\hline $\begin{array}{c}\mathrm{Te} \\
0.14\end{array}$ & $\begin{array}{c}0.23(0.19-0.27) \\
0.25 \pm 0.09 \\
0.21(0.14)\end{array}$ & $\begin{array}{c}0.23(0.19-0.28) \\
0.27 \pm 0.17 \\
0.21(0.24)\end{array}$ & $\begin{array}{c}1.01 \\
(0.78-1.32)\end{array}$ & 0.9417 \\
\hline $\begin{array}{c}\mathrm{Ba} \\
1.86\end{array}$ & $\begin{array}{c}1.27(0.94-1.72) \\
1.61 \pm 1.19 \\
1.16(0.74)\end{array}$ & $\begin{array}{c}1.65(1.04-2.57) \\
3.54 \pm 4.96 \\
1.89(3.98)\end{array}$ & $\begin{array}{c}0.77 \\
(0.45-1.33)\end{array}$ & 0.3442 \\
\hline $\begin{array}{c}\mathrm{Tl} \\
0.18\end{array}$ & $\begin{array}{c}0.14(0.11-0.17) \\
0.16 \pm 0.11 \\
0.13(0.05)\end{array}$ & $\begin{array}{c}0.23(0.18-0.29) \\
0.29 \pm 0.19 \\
0.25(0.16)\end{array}$ & $\begin{array}{c}0.59 \\
(0.42-0.83)\end{array}$ & 0.0031 \\
\hline $\begin{array}{c}\mathrm{Pb} \\
1.78\end{array}$ & $\begin{array}{c}1.76(1.38-2.26) \\
2.09 \pm 1.28 \\
1.68(1.28)\end{array}$ & $\begin{array}{c}1.24(0.90-1.69) \\
1.91 \pm 2.50 \\
1.24(1.22)\end{array}$ & $\begin{array}{c}1.42 \\
(0.95-2.15)\end{array}$ & 0.0888 \\
\hline $\begin{array}{c}\mathrm{U} \\
<0.007\end{array}$ & $\begin{array}{c}0.008(0.006-0.0105) \\
0.11 \pm 0.01 \\
0.007(0.004)\end{array}$ & $\begin{array}{c}0.02(0.016-0.031) \\
0.03 \pm 0.04 \\
0.02(0.03)\end{array}$ & $\begin{array}{c}0.37 \\
(0.24-0.55)\end{array}$ & $<0.0001$ \\
\hline
\end{tabular}

DCM prediction models with adjustment for age, sex, or educational level showed that higher levels of chromium, copper, zinc, selenium, cadmium, antimony, thallium, and uranium in urine were significantly associated with DCM (Table 4). At concentrations (in $\mu \mathrm{g} / \mathrm{g}$ ) of creatinine above 1 for chromium, 20 for copper, 600 for zinc, 30 for selenium, 2 for cadmium, 0.2 for antimony, 0.5 for thallium, and 0.05 for uranium, the probability of DCM was almost maximum (Figure 3). Significant correlations were seen between blood and urinary concentrations of several of the heavy metals of interest (Table S3 and Figure S1). 


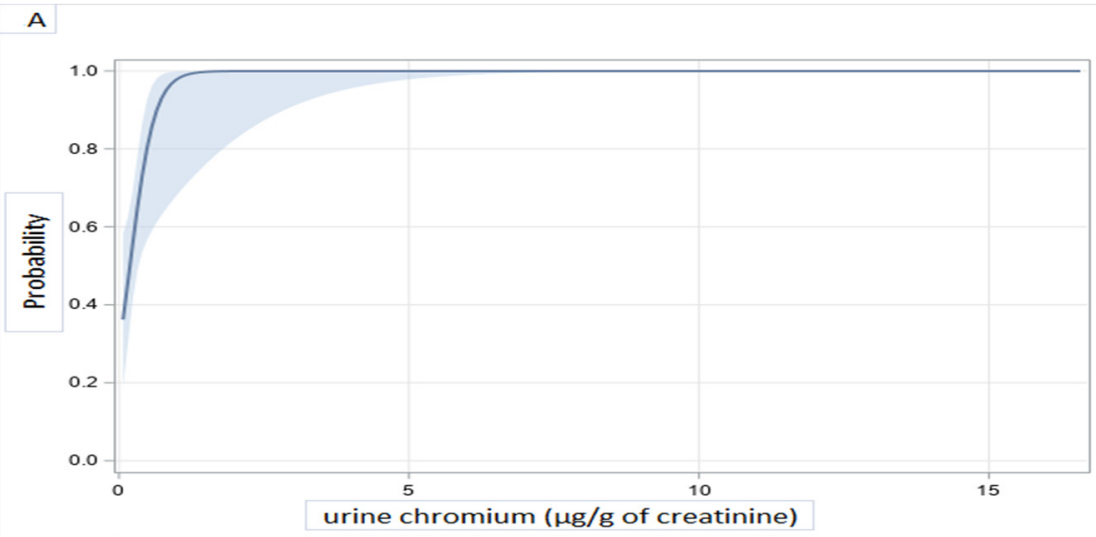

B

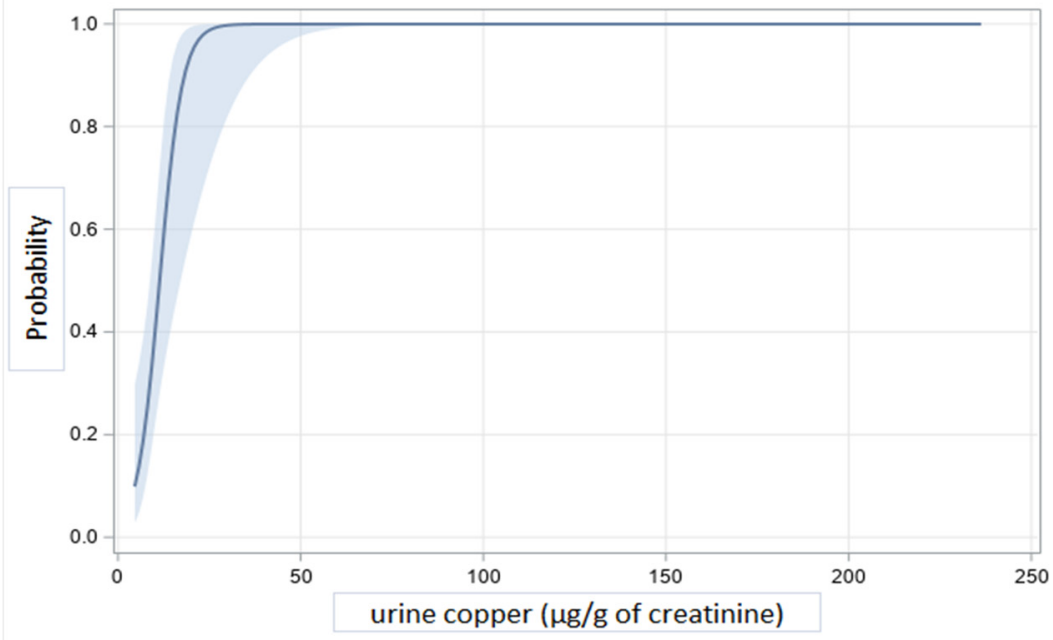

C

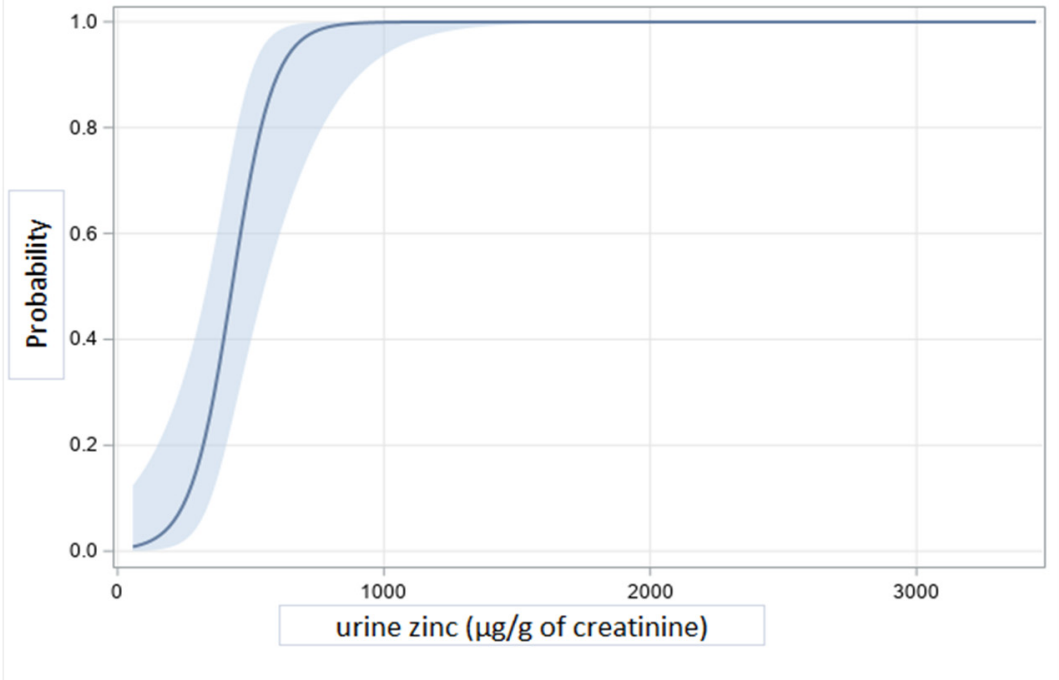

Figure 3. Cont. 
D

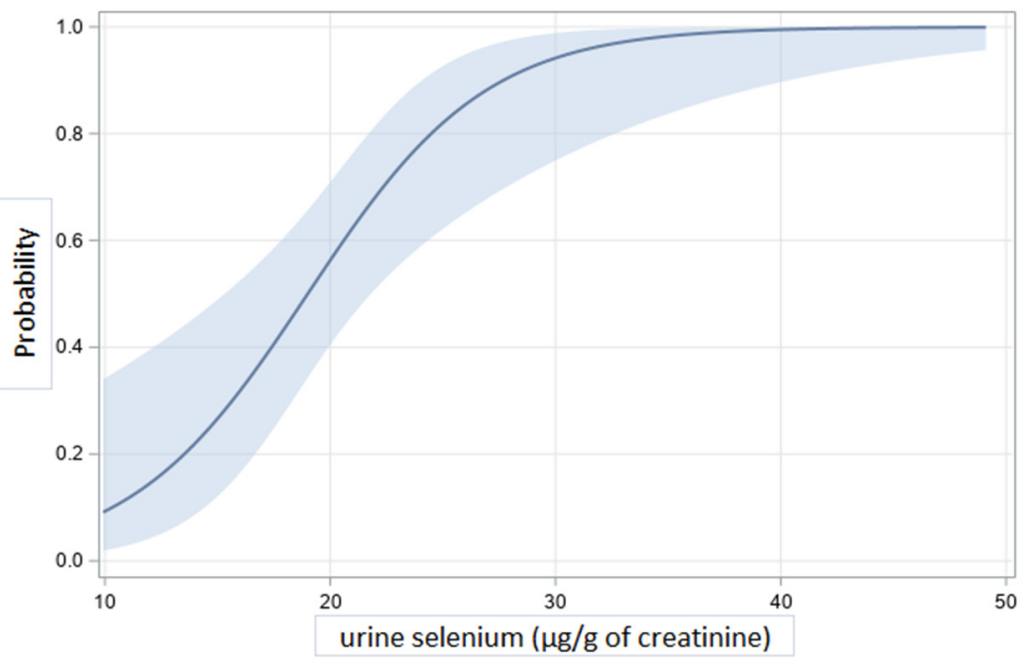

E

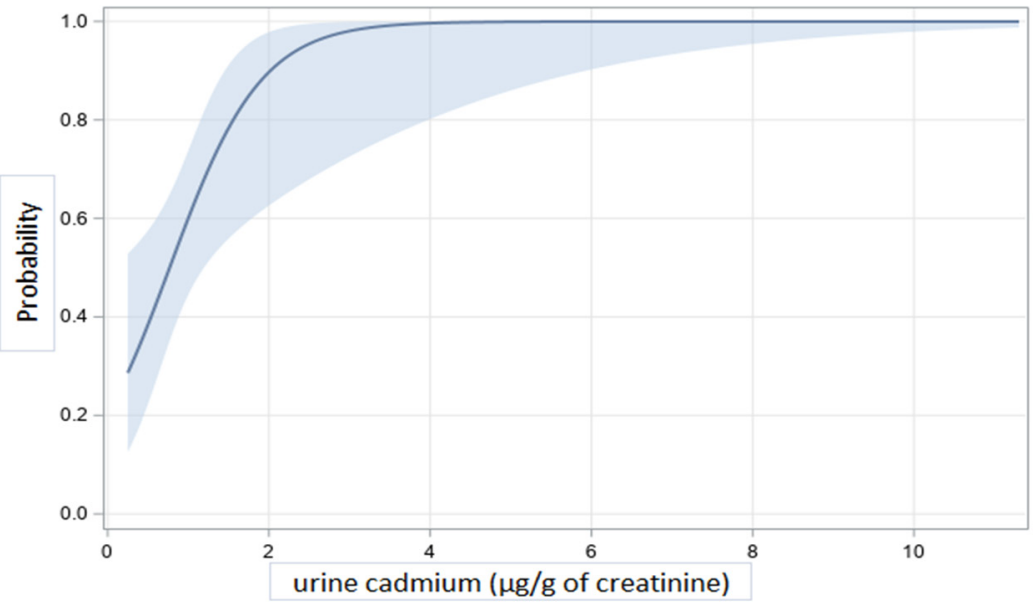

$\mathrm{F}$

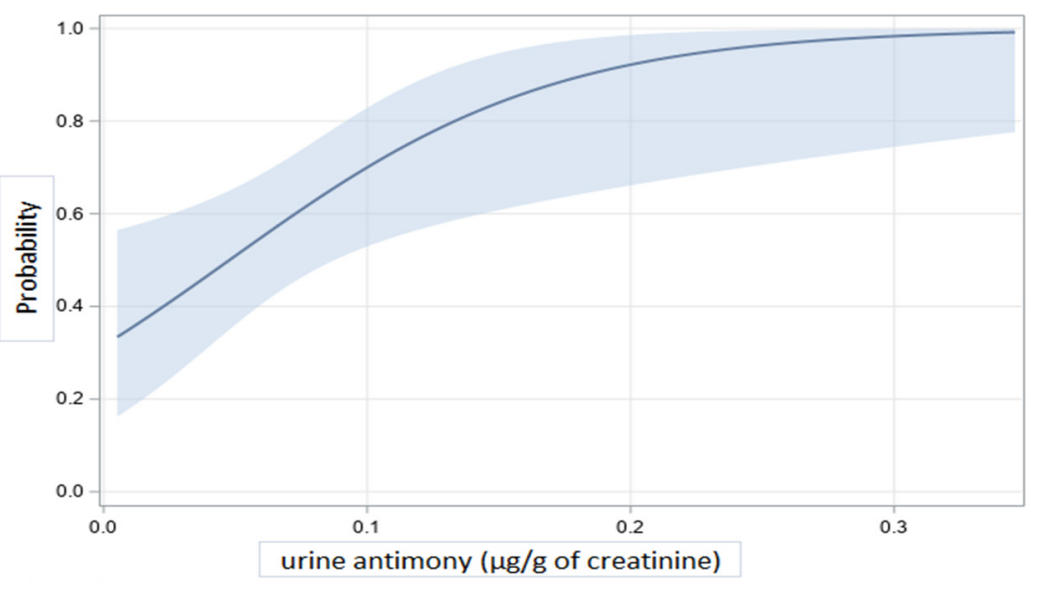

Figure 3. Cont. 


\section{G}

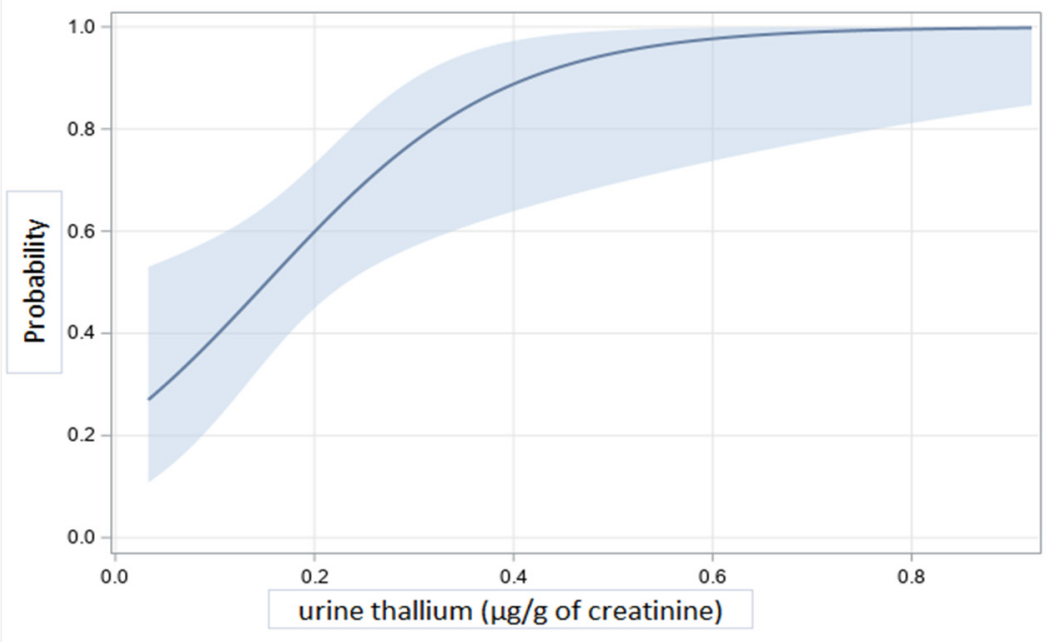

$\mathrm{H}$

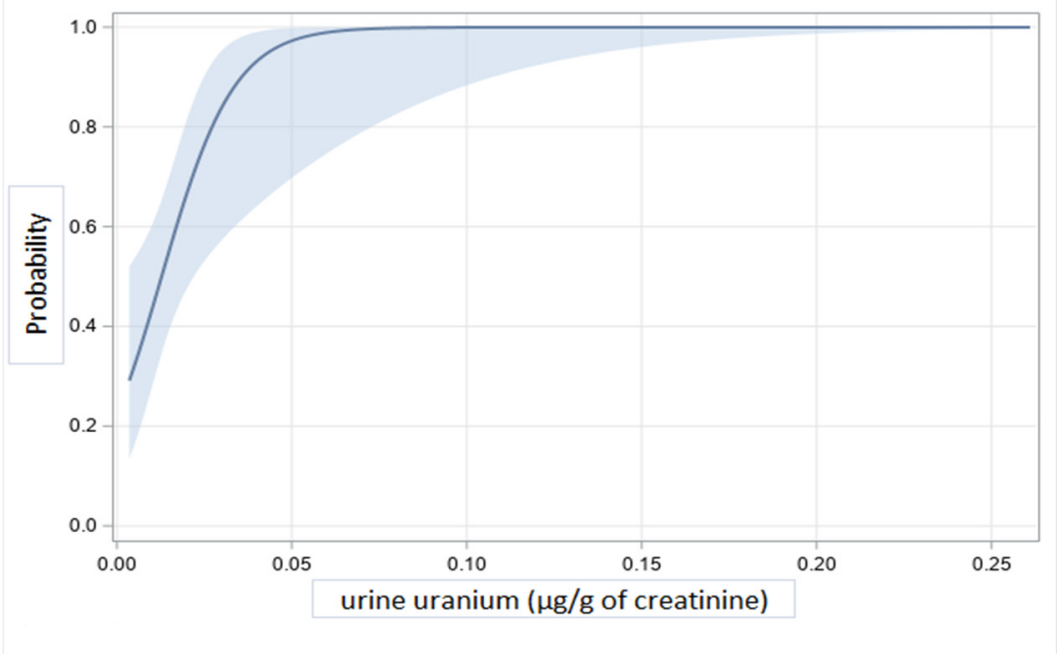

Figure 3. Illustrative predicted probabilities of dilated cardiomyopathy as a function of heavy metals concentrations in urine: chromium (A), copper (B), zinc (C), selenium (D), cadmium (E), antimony $(\mathbf{F})$, thallium $(\mathbf{G})$, and uranium $(\mathbf{H})$. 
Table 4. Adjusted models of DCM prediction by urine metal concentrations $(N=57)$.

\begin{tabular}{|c|c|c|c|c|c|c|}
\hline Variable & Coefficient & SE & \multicolumn{2}{|c|}{ Wald 95\% Confidence Limits } & Wald $X^{2}$ & $p$-Value \\
\hline \multicolumn{7}{|c|}{ Chromium model } \\
\hline Intercept & -2.32 & 1.29 & -4.85 & 0.21 & 3.24 & 0.0717 \\
\hline Chromium & 4.69 & 2.09 & 0.60 & 8.78 & 5.05 & 0.0246 \\
\hline Age & 0.02 & 0.02 & -0.02 & 0.07 & 0.90 & 0.3435 \\
\hline Male sex & 1.04 & 0.69 & -0.32 & 2.40 & 2.24 & 0.1344 \\
\hline Education X & -0.86 & 0.65 & -2.13 & 0.41 & 1.76 & 0.1847 \\
\hline \multicolumn{7}{|c|}{ Copper model } \\
\hline Intercept & -5.87 & 2.15 & -10.10 & -1.65 & 7.44 & 0.0064 \\
\hline Copper & 0.32 & 0.11 & 0.10 & 0.54 & 8.32 & 0.0039 \\
\hline Age & 0.03 & 0.04 & -0.04 & 0.10 & 0.69 & 0.4058 \\
\hline Male sex & 0.88 & 0.95 & -0.98 & 2.74 & 0.85 & 0.3556 \\
\hline Education & 0.08 & 0.94 & -1.76 & 1.93 & 0.01 & 0.9313 \\
\hline \multicolumn{7}{|c|}{ Zinc model } \\
\hline Intercept & -4.88 & 2.65 & -10.07 & 0.29 & 3.41 & 0.0648 \\
\hline Zinc & 0.02 & 0.01 & 0.01 & 0.03 & 9.45 & 0.0021 \\
\hline Age & -0.05 & 0.06 & -0.17 & 0.07 & 0.67 & 0.4146 \\
\hline Male sex & -0.81 & 1.26 & -3.28 & 1.67 & 0.41 & 0.5231 \\
\hline Education & 0.95 & 1.31 & -1.62 & 3.52 & 0.53 & 0.4684 \\
\hline \multicolumn{7}{|c|}{ Selenium model } \\
\hline Intercept & -7.39 & 2.41 & -12.13 & -2.66 & 9.38 & 0.0022 \\
\hline Selenium & 0.24 & 0.08 & 0.09 & 0.39 & 9.84 & 0.0017 \\
\hline Age & 0.05 & 0.03 & -0.01 & 0.10 & 3.11 & 0.0780 \\
\hline Male sex & 1.51 & 0.78 & -0.02 & 3.05 & 3.74 & 0.0533 \\
\hline High education & -1.28 & 0.74 & -2.73 & 0.16 & 3.03 & 0.0818 \\
\hline \multicolumn{7}{|c|}{ Cadmium model } \\
\hline Intercept & -2.19 & 1.35 & -4.83 & 0.46 & 2.63 & 0.1051 \\
\hline Cadmium & 2.47 & 0.87 & 0.78 & 4.17 & 8.19 & 0.0042 \\
\hline Age & -0.01 & 0.03 & -0.07 & 0.05 & 0.16 & 0.6850 \\
\hline Male sex & 1.39 & 0.77 & -0.11 & 2.91 & 3.29 & 0.0697 \\
\hline Education & -0.83 & 0.70 & -2.20 & 0.55 & 1.38 & 0.2401 \\
\hline \multicolumn{7}{|c|}{ Antimony model } \\
\hline Intercept & -2.39 & 1.33 & -4.99 & -0.22 & 3.23 & 0.0723 \\
\hline Antimony & 14.59 & 6.11 & 2.61 & 26.56 & 5.70 & 0.0170 \\
\hline Age & 0.03 & 0.02 & -00.1 & 0.08 & 1.97 & 0.1602 \\
\hline Male sex & 0.56 & 0.64 & -0.69 & 1.82 & 0.78 & 0.3785 \\
\hline Education & -0.77 & 0.65 & -2.04 & 0.51 & 1.39 & 0.2380 \\
\hline \multicolumn{7}{|c|}{ Thallium model } \\
\hline Intercept & -3.13 & 1.40 & -5.88 & -0.38 & 4.96 & 0.0259 \\
\hline Thallium & 8.51 & 3.32 & 2.01 & 15.01 & 6.58 & 0.0103 \\
\hline Age & 0.03 & 0.03 & -0.02 & 0.08 & 1.43 & 0.2324 \\
\hline Male sex & 1.36 & 0.74 & -0.08 & 2.80 & 3.42 & 0.0645 \\
\hline Education & -1.25 & 0.69 & -2.59 & 0.09 & 3.33 & 0.0681 \\
\hline \multicolumn{7}{|c|}{ Uranium model } \\
\hline Intercept & -3.78 & 1.62 & -6.95 & -0.61 & 5.48 & 0.0193 \\
\hline Uranium & 85.43 & 35.37 & 16.11 & 154.74 & 5.83 & 0.0157 \\
\hline Age & 0.04 & 0.03 & -0.01 & 0.09 & 2.62 & 0.1055 \\
\hline Male sex & 1.59 & 0.78 & 0.07 & 3.10 & 4.19 & 0.0408 \\
\hline Education & -0.92 & 0.71 & -2.32 & 0.47 & 1.68 & 0.1944 \\
\hline
\end{tabular}

X: Being highly educated (university or post-university) often correlates with higher socio-economic status in the KC settings. 


\section{Discussion}

This is the first study to look at estimated Glomerular Filtration Rate (eGFR)-adjusted associations between idiopathic DCM and high blood or urinary concentrations of several heavy metals in a rarely documented context of concurrent and multiple exposures. Blood and urinary concentrations for most of the metals of interest were above reference values $[38,44,47]$. In addition, significant correlations were found between blood and urine concentrations of metals, underlining complex interactions in the biology of heavy metals including those with known beneficial biological properties strengthening the validity of study procedures and measurements [48,49].

Blood and/or urinary concentrations of several metals were above reference values both in subjects with DCM and those presumably healthy though significantly higher in subjects with DCM when considering metal concentrations in urine. In the DRC contexts of poorly regulated mining and consistent with previous studies [14,19,38], our findings possibly reflect community-wide concurrent and multiple exposures to toxic compounds. We also showed that DCM probability almost reaches the maximum beyond specific thresholds for blood arsenic and copper concentrations or urinary concentrations of chromium, cadmium, antimony, thallium, and uranium.

The blood arsenic concentration found in our subjects $(0.39 \mu \mathrm{g} / \mathrm{dL})$ far exceeds what has been reported in the general population of France $(0.17 \mu \mathrm{g} / \mathrm{dL})$ [47], Brazil $(0.11 \mu \mathrm{g} / \mathrm{dL})$ [50], Pakistan $(0.21 \mu \mathrm{g} / \mathrm{dL})$ [51], and China $(0.23 \mu \mathrm{g} / \mathrm{dL})$ [52]. Whether the association between DCM probability and higher blood levels of arsenic implies the later may contribute to the etiology of DCM is not known, but remains a possibility. Experimental studies in rodents, however, have demonstrated the toxic effect of arsenic on myocardial tissue through the inhibition of anti-oxidative stress defense enzymes [53,54]. Arsenic exposure has been associated with cardiopathologic effects, including ischemia, arrhythmia, and heart failure [55]. Possible mechanisms include increased oxidative stress, depletion of antioxidant status, DNA fragmentation, apoptosis by mitochondrial disruption, caspase activation, MAPK signaling and p53, functional changes in ion channels, and dyshomeostasis of trace elements [55]. It has also been revealed that arsenic can induce all kinds of diseases, including heart diseases through epigenetic modifications associated with hypermethylation of genes coding for ion channels and diverse proteins of oxidative stress and energy production [56,57].

Exposure to copper is associated with increased cardiovascular risk [58]. High copper concentrations had been associated with heart failure incidence [36,59-63] and bad outcomes such as re-hospitalizations and deaths over a one-year follow-up [64]. Copper is an important trace element in humans that is incorporated in several enzymes of vital functions. However, at high concentrations due to permanent exposure or in Wilson's disease, for example, its free fraction increases in such manner that its infiltrates the myocardial tissue, induces cellular toxicity, and promotes harmful free radical formation [65-68]. Although the blood concentration of copper reported in this study was slightly higher than the upper limit of the reference value (143.7 vs. $140 \mu \mathrm{g} / \mathrm{dL}$ ) [69], the urine concentration far exceeds levels reported as reference value $[44,70]$ and in a very high-exposure environment [38]. This high urinary concentration, which, moreover, was strongly correlated with blood concentration $(r=0.73235, p<0.000)$, was precisely such an indication of a strong recent exposure. It was also an indication of a permanent exposure to this metal, which, ironically, is the great wealth of the KC. Because of copper and cobalt, the $\mathrm{KC}$ has been invaded by many mining companies that are the main polluters in the region.

The association between zinc deficiency and dilated cardiomyopathy is well documented $[45,59,60,71-73]$. Urinary concentrations of $\mathrm{Zn}$ in subjects with DCM was 4 times the reference value denoting very high excretion [47], a commonly noted phenomenon during heart failure. Aldosteronism and activation of atrial natriuretic peptides increase urinary excretion of zinc and loss in feces [74,75]. Aldosteronism also causes intracellular calcium overload, with the consequent induction of oxidative stress leading to necrosis of myocardial cells. Increasing intracellular calcium is coupled with zinc entry into cells to 
counteract the prooxidant effect of calcium overload. Aldosteronism causes acidification of the urine and a state of alkalosis, which are conducive to the urinary excretion of zinc. The inverse effects of acetazolamide would therefore be beneficial to curb this excretion [76]. Competition with heavy metals, especially with copper and cadmium, also explains its blood deficit and high urine excretion [77]. As zinc serves to maintain normal cell structure and function by its anti-oxidant and anti-inflammatory properties [71,78], both absolute and relative deficiency would impact negatively the proper function of the heart.

We found a strong correlation between blood copper and urinary zinc that illustrated the intimate relationship between the two metals. Indeed, the increase in blood copper leads to increased excretion of zinc and consequently its deficit. The copper-zinc intimacy is also illustrated by the $\mathrm{Zn} / \mathrm{Cu}$ ratio. The fact that the highest $\mathrm{Zn} / \mathrm{Cu}$ ratios were associated with a zero probability of DCM is in line with previous findings [62].

Urinary levels of chromium, cadmium, antimony, thallium, and uranium were significantly higher in DCM patients than controls. These toxic metals have all been implicated in cardiovascular disease. For example, the concept of chromium cardiomyopathy is discussed in a study in rats that were treated with potassium dichromate, and later showed changes in cardiac muscle and interstitial fibrosis. In addition, there was vacuolization, hemorrhages, and cell necrosis [79]. Accumulation of chromium in the myocardial tissue of patients with idiopathic DCM [33] has also been reported. Cadmium exposure may increase the prevalence of stroke and heart failure [80]. At lower concentrations than those of this study, cadmium was associated with idiopathic dilated cardiomyopathy [81]. These cadmium concentrations being obtained in essentially non-smoking patients argues in favor of exogenous (environmental) exposure.

Leishmaniasis treatment with antimony can cause serious arrhythmias such as torsadede-pointe or, reportedly, an alteration of myocardial contractility through oxidative stress and disruption of intracellular calcium handling $[34,35,82,83]$. In our study, higher urinary concentrations of antimony were associated with DCM, supporting the above thesis on putative cardiotoxicity, and this was possibly due to yet-to-be-fully-documented environmental exposures.

Thallium, an extremely toxic metal, has the same ionic radius as potassium. Thallium follows the distribution of potassium in the cellular and extracellular compartments. The cell membrane cannot differentiate between these two ions. The Na+/K+-ATPase pump has ten times more affinity for thallium than for potassium. Thus, all potassium-dependent biological processes are altered in the presence of thallium. Through this, thallium can have immediate cardiovascular effects such as tachycardia, hypertension, ventricular fibrillation, and other electrocardiographic abnormalities. Thallium can also directly stimulate chromaffin cells and lead to a significant release of catecholamines that are deleterious for the myocardium [84-87]. Measurement of urinary thallium is a simple way to detect exposure to thallium [88]. The fact that thallium was found in greater concentration in the patients' urine relative to controls suggests a participation of this metal in DCM morbidity.

Known studies in animal models exposed to uranium have not shown a cardiovascular effect $[89,90]$. However, numerous signs have been reported in the course of acute uranium poisoning, including myocarditis resulting in episodic atrial flutter [91]. Patients' urinary uranium was approximately 2.5 times that of controls and reference values. This may suggest a harmful effect of this metal on the myocardium.

It is difficult to determine whether blood and/or urinary concentrations of all the above culprits contribute to DCM morbidity due to challenges in analytical methods for multiple exposures and because of mutual influences heavy metals exert on each other biology in concurrent exposures. A relatively small sample size and the lack of normal reference for the $\mathrm{KC}$ population are the main limitations of the present study. A larger sample size would have allowed studying the interactions between metals and controlling for other important clinical and/or biological features including but not limited to inflammation and/or liver functions. Nevertheless, our findings are consistent with those from previous studies that have shown ubiquitous and concurrent exposures to potentially 
cardiotoxic metals in the KC mining area. GFR-adjusted associations between DCM and concentrations of several of the above-mentioned heavy metals warrant public health measures to mitigate exposures to the toxic culprits. In addition, we recommend screening for heavy metals in contexts of cardiac morbidity and obvious environmental pollution from mining and/or ore processing activities. Chelation therapies and/or supplementation with elements with beneficial benefits to cellular homeostasis should also be thoroughly assessed as direct options to prevent cardiotoxicity in such contexts.

Supplementary Materials: The following are available online at https: / www.mdpi.com/article / 10.3390/ijerph18094956/s1, Figure S1: Illustrative correlations between concentrations of copper or arsenic in the blood and concentrations of zinc, selenium, cadmium, and copper in the urine., Table S1: Clinical features of subjects with dilated cardiomyopathy, Table S2: Comparison of heart failure patients to controls according to demography, education level, and routine biology, Table S3: Correlations between blood and urine metal concentrations.

Author Contributions: D.M.-L., D.T.-K., and D.N.-N. designed the study; D.M.-L., D.N.-N. performed the measurements; D.N.-N. and P.K. were involved in planning and supervised the work; D.M.-L. performed the analysis, drafted the manuscript, and designed the figures. D.T.-K., V.B., J.-M.R., R.K.K., E.N.Y., P.K., B.K.-B. aided in interpreting the results and finalizing the manuscript. All authors have read and agreed to the published version of the manuscript.

Funding: This research was funding by Vlaamse Interuniversitaire Raad (CD2017TEA439A104) and University of Hasselt; supported in part by National Institutes of Health grant NIEHS/FIC R01ES019841 for training and capacity building.

Informed Consent Statement: Informed consent was obtained from all subjects involved in the study.

Data Availability Statement: The study was conducted according to the guidelines of the Declaration of Helsinki, and was approved on 9 August 2017 by the Committee of Medical Ethics of the University of Lubumbashi (UNILU/CEM/075/2017).

Availability of Data and Materials: The fully anonymized data that support the findings of this study are available in the database of the Department of Internal Medicine of the Faculty of Medicine of University of Lubumbashi.

Acknowledgments: We are grateful to Wilfried Mullens for his fruitful comments on the research work and findings. We thank Célestin Banza Lubaba Nkulu, who facilitated the storage and shipment of samples in Belgium for the ICP-MS metal assay; Benoît Nemery, for his involvement so that our samples can be analyzed in priority at the UCL laboratory; and all patients and controls who understood the merits of this study and who gave their consent to be participants. We also thank Denk Pharma for the drugs provided free of charge to patients during the study.

Conflicts of Interest: The authors declare no conflict of interest.

\section{References}

1. Ober, J.A. Mineral Commodity Summaries 2018; Geological Survey: Liston, VA, USA, 2018.

2. Cailteux, J.L.H.; Kampunzu, A.B.; Lerouge, C.; Kaputo, A.K.; Milesi, J.P. Genesis of sediment-hosted stratiform copper-cobalt deposits, central African Copperbelt. J. Afr. Earth Sci. 2005, 42, 134-158. [CrossRef]

3. Sovacool, B.K. The precarious political economy of cobalt: Balancing prosperity, poverty, and brutality in artisanal and industrial mining in the Democratic Republic of the Congo. Extr. Ind. Soc. 2019. [CrossRef]

4. Pourret, O.; Lange, B.; Bonhoure, J.; Colinet, G.; Decrée, S.; Mahy, G.; Séleck, M.; Shutcha, M.; Faucon, M.-P. Assessment of soil metal distribution and environmental impact of mining in Katanga (Democratic Republic of Congo). Appl. Geochem. 2016, 64, 43-55. [CrossRef]

5. Prasad, M.S. Production of copper and cobalt at Gecamines, Zaire. Miner. Eng. 1989, 2, 521-541. [CrossRef]

6. Edwards, D.P.; Sloan, S.; Weng, L.; Dirks, P.; Sayer, J.; Laurance, W.F. Mining and the African Environment. Conserv. Lett. 2014, 7, 302-311. [CrossRef]

7. Atibu, E.K.; Lacroix, P.; Sivalingam, P.; Ray, N.; Giuliani, G.; Mulaji, C.K.; Otamonga, J.P.; Mpiana, P.T.; Slaveykova, V.I.; Pote, J. High contamination in the areas surrounding abandoned mines and mining activities: An impact assessment of the Dilala, Luilu and Mpingiri Rivers, Democratic Republic of the Congo. Chemosphere 2018, 191, 1008-1020. [CrossRef] 
8. Atibu, E.K.; Devarajan, N.; Thevenon, F.; Mwanamoki, P.M.; Tshibanda, J.B.; Mpiana, P.T.; Prabakar, K.; Mubedi, J.I.; Wildi, W.; Poté, J. Concentration of metals in surface water and sediment of Luilu and Musonoie Rivers, Kolwezi-Katanga, Democratic Republic of Congo. Appl. Geochem. 2013, 39, 26-32. [CrossRef]

9. Muhaya, B.B.; wa Kayembe, M.K.; Kunyonga, C.Z.; Catherine, S. Assessment of Trace Metal Contamination of Sediments in the Lubumbashi River Basin, Kafubu, Kimilolo and Kinkalabwamba Rivers in Lubumbashi City, Democratic Republic of Congo. J. Environ. Sci. Eng. A 2017, 6, 167-177.

10. Muhaya, B.B.; Kunyonga, C.Z.; Mulongo, S.C.; Mushobekwa, F.Z.; Bisimwa, A.M. Trace Metal Contamination of Sediments in Naviundu River Basin, Luano and Ruashi Rivers and Luwowoshi Spring in Lubumbashi City, Democratic Republic of Congo. J. Environ. Sci. Eng. B 2017. [CrossRef]

11. Squadrone, S.; Burioli, E.; Monaco, G.; Koya, M.K.; Prearo, M.; Gennero, S.; Dominici, A.; Abete, M.C. Human exposure to metals due to consumption of fish from an artificial lake basin close to an active mining area in Katanga (D.R. Congo). Sci. Total Environ. 2016, 568, 679-684. [CrossRef]

12. Cheyns, K.; Banza Lubaba Nkulu, C.; Ngombe, L.K.; Asosa, J.N.; Haufroid, V.; De Putter, T.; Nawrot, T.; Kimpanga, C.M.; Numbi, O.L.; Ilunga, B.K.; et al. Pathways of human exposure to cobalt in Katanga, a mining area of the D.R. Congo. Sci. Total Environ. 2014, 490, 313-321. [CrossRef] [PubMed]

13. Mudimbi Kalonda, D.; Kabamba Tshikongo, A.; Kodondi Kule Koto, F.; Kasongo Busambwa, C.; Kisunka Bwalya, Y.; Musola Cansa, H.; Kahambwe Tambwe, J.-L.; Lukumwena Kalala, Z.; Longanga Otshudi, A. Profile of heavy metals contained in food plants commonly consumed in a few mining areas of Katanga province. J. Appl. Biosci. 2015, 96, 9049-9054. [CrossRef]

14. Banza, C.L.; Nawrot, T.S.; Haufroid, V.; Decree, S.; De Putter, T.; Smolders, E.; Kabyla, B.I.; Luboya, O.N.; Ilunga, A.N.; Mutombo, A.M.; et al. High human exposure to cobalt and other metals in Katanga, a mining area of the Democratic Republic of Congo. Environ. Res. 2009, 109, 745-752. [CrossRef] [PubMed]

15. Narendrula, R.; Nkongolo, K.K.; Beckett, P. Comparative soil metal analyses in Sudbury (Ontario, Canada) and Lubumbashi (Katanga, DR-Congo). Bull. Environ. Contam. Toxicol. 2012, 88, 187-192. [CrossRef] [PubMed]

16. Atibu, E.K.; Devarajan, N.; Laffite, A.; Giuliani, G.; Salumu, J.A.; Muteb, R.C.; Mulaji, C.K.; Otamonga, J.-P.; Elongo, V.; Mpiana, P.T.; et al. Assessment of trace metal and rare earth elements contamination in rivers around abandoned and active mine areas. The case of Lubumbashi River and Tshamilemba Canal, Katanga, Democratic Republic of the Congo. Chem. Der Erdegeochem. 2016, 76, 353-362. [CrossRef]

17. Nemery, B.; Banza Lubaba Nkulu, C. Assessing exposure to metals using biomonitoring: Achievements and challenges experienced through surveys in low- and middle-income countries. Toxicol. Lett. 2018, 298, 13-18. [CrossRef] [PubMed]

18. Mukendi, R.A.; Banza, C.L.N.; Mukeng, C.A.; Ngwe, J.T.M.; Mwembo, A.N.; Kalenga, P.M.K. Exposure of man to metal trace elements and alteration of sperm parameters: Study conducted in the mining areas of Haut-Katanga in the Democratic Republic of Congo. Pan Afr. Med. J. 2018, 30, 35. [CrossRef]

19. Bora, B.K.; Ramos-Crawford, A.L.; Sikorskii, A.; Boivin, M.J.; Lez, D.M.; Mumba-Ngoyi, D.; Mukalay Wa Mukalay, A.; OkitunduLuwa, D.; Tshala-Katumbay, D. Concurrent exposure to heavy metals and cognition in school-age children in Congo-Kinshasa: A complex overdue research agenda. Brain Res. Bull. 2019, 145, 81-86. [CrossRef]

20. Mbuyi-Musanzayi, S.; Kayembe, T.J.; Kashal, M.K.; Lukusa, P.T.; Kalenga, P.M.; Tshilombo, F.K.; Devriendt, K.; Reychler, H. Non-syndromic cleft lip and/or cleft palate: Epidemiology and risk factors in Lubumbashi (DR Congo), a case-control study. J. Cranio-Maxillo-Facial Surg. 2018, 46, 1051-1058. [CrossRef] [PubMed]

21. Lubala, T.K.; Shongo, M.Y.; Munkana, A.N.; Mutombo, A.M.; Mbuyi, S.M.; wa Momat, F.K. Congenital malformations in Lubumbashi (Democratic Republic of Congo): About 72 cases observed and advocacy for the development of a National Registry of Congenital Malformations and a National Reference Center for Human Genetics. Pan Afr. Med. J. 2012, 13, 84. [CrossRef]

22. Kayembe-Kitenge, T.; Kasole Lubala, T.; Musa Obadia, P.; Katoto Chimusa, P.; Katshiez Nawej, C.; Banza Lubaba Nkulu, C.; Devriendt, K.; Nemery, B. Holoprosencephaly: A case series from an area with high mining-related pollution. Birth Defects Res. 2019, 111, 1561-1563. [CrossRef] [PubMed]

23. Chowdhury, R.; Ramond, A.; O’Keeffe, L.M.; Shahzad, S.; Kunutsor, S.K.; Muka, T.; Gregson, J.; Willeit, P.; Warnakula, S.; Khan, H.; et al. Environmental toxic metal contaminants and risk of cardiovascular disease: Systematic review and meta-analysis. BMJ 2018, 362, k3310. [CrossRef] [PubMed]

24. Aneni, E.C.; Escolar, E.; Lamas, G.A. Chronic Toxic Metal Exposure and Cardiovascular Disease: Mechanisms of Risk and Emerging Role of Chelation Therapy. Curr. Atheroscler. Rep. 2016, 18, 1-8. [CrossRef] [PubMed]

25. Tellez-Plaza, M.; Jones, M.R.; Dominguez-Lucas, A.; Guallar, E.; Navas-Acien, A. Cadmium exposure and clinical cardiovascular disease: A systematic review. Curr. Atheroscler. Rep. 2013, 15, 356. [CrossRef] [PubMed]

26. Ferreira de Mattos, G.; Costa, C.; Savio, F.; Alonso, M.; Nicolson, G.L. Lead poisoning: Acute exposure of the heart to lead ions promotes changes in cardiac function and Cav1.2 ion channels. Biophys. Rev. 2017, 9, 807-825. [CrossRef] [PubMed]

27. Xie, J.; Du, G.; Zhang, Y.; Zhou, F.; Wu, J.; Jiao, H.; Li, Y.; Chen, Y.; Ouyang, L.; Bo, D.; et al. ECG conduction disturbances and ryanodine receptor expression levels in occupational lead exposure workers. Occup. Environ. Med. 2019, 76, 151-156. [CrossRef]

28. Shen, J.; Wang, X.; Zhou, D.; Li, T.; Tang, L.; Gong, T.; Su, J.; Liang, P. Modelling cadmium-induced cardiotoxicity using human pluripotent stem cell-derived cardiomyocytes. J. Cell. Mol. Med. 2018, 22, 4221-4235. [CrossRef] [PubMed]

29. Chen, C.Y.; Zhang, S.L.; Liu, Z.Y.; Tian, Y.; Sun, Q. Cadmium toxicity induces ER stress and apoptosis via impairing energy homoeostasis in cardiomyocytes. Biosci. Rep. 2015, 35. [CrossRef] 
30. Ferramola, M.L.; Pérez Díaz, M.F.F.; Honoré, S.M.; Sánchez, S.S.; Antón, R.I.; Anzulovich, A.C.; Giménez, M.S. Cadmium-induced oxidative stress and histological damage in the myocardium. Effects of a soy-based diet. Toxicol. Appl. Pharmacol. 2012, 265, 380-389. [CrossRef]

31. Furieri, L.B.; Fioresi, M.; Junior, R.F.R.; Bartolomé, M.V.; Fernandes, A.A.; Cachofeiro, V.; Lahera, V.; Salaices, M.; Stefanon, I.; Vassallo, D.V. Exposure to low mercury concentration in vivo impairs myocardial contractile function. Toxicol. Appl. Pharmacol. 2011, 255, 193-199. [CrossRef]

32. Lorscheider, F.; Vimy, M. Mercury and idiopathic dilated cardiomyopathy. J. Am. Coll. Cardiol. 2000, 35, 819. [CrossRef]

33. Frustaci, A.; Magnavita, N.; Chimenti, C.; Caldarulo, M.; Sabbioni, E.; Pietra, R.; Cellini, C.; Possati, G.F.; Maseri, A. Marked elevation of myocardial trace elements in idiopathic dilated cardiomyopathy compared with secondary cardiac dysfunction. $J$. Am. Coll. Cardiol. 1999, 33, 1578-1583. [CrossRef]

34. Hantson, P. Mechanisms of toxic cardiomyopathy. Clin. Toxicol. 2018, 57, 1-9. [CrossRef] [PubMed]

35. Perez, A.L.; Tang, W.H. Contribution of environmental toxins in the pathogenesis of idiopathic cardiomyopathies. Curr. Treat. Options Cardiovasc. Med. 2015, 17, 381. [CrossRef]

36. Alexanian, I.; Parissis, J.; Farmakis, D.; Athanaselis, S.; Pappas, L.; Gavrielatos, G.; Mihas, C.; Paraskevaidis, I.; Sideris, A.; Kremastinos, D.; et al. Clinical and echocardiographic correlates of serum copper and zinc in acute and chronic heart failure. Clin. Res. Cardiol. 2014, 103, 938-949. [CrossRef] [PubMed]

37. Malamba-Lez, D.; Ngoy-Nkulu, D.; Steels, P.; Tshala-Katumbay, D.; Mullens, W. Heart Failure Etiologies and Challenges to Care in the Developing World: An Observational Study in the Democratic Republic of Congo. J. Card Fail. 2018, 24, 854-859. [CrossRef]

38. Banza Lubaba Nkulu, C.; Casas, L.; Haufroid, V.; De Putter, T.; Saenen, N.D.; Kayembe-Kitenge, T.; Musa Obadia, P.; Kyanika Wa Mukoma, D.; Lunda Ilunga, J.M.; Nawrot, T.S.; et al. Sustainability of artisanal mining of cobalt in DR Congo. Nat. Sustain. 2018, 1, 495-504. [CrossRef]

39. Muhaya, B.B.; Mulongo, S.C.; Kunyonga, C.Z.; Mushobekwa, F.Z.; Wa Kayembe, M.K. Trace Metal Contamination of Water in Naviundu River Basin, Luano and Ruashi Rivers and Luwowoshi Spring in Lubumbashi City, Democratic Republic of Congo. J. Environ. Sci. Eng. A 2017, 6, 329-336.

40. Initiative EEIT. The Democratic Republic of Congo. 2019. Available online: https://eiti.org/democratic-republic-of-congo (accessed on 17 July 2019).

41. Lang, R.M.; Badano, L.P.; Mor-Avi, V.; Afilalo, J.; Armstrong, A.; Ernande, L.; Flachskampf, F.A.; Foster, E.; Goldstein, S.A.; Kuznetsova, T.; et al. Recommendations for cardiac chamber quantification by echocardiography in adults: An update from the American Society of Echocardiography and the European Association of Cardiovascular Imaging. Eur. Heart J. Cardiovasc. Imaging 2015, 16, 233-270. [CrossRef]

42. Nagueh, S.F.; Smiseth, O.A.; Appleton, C.P.; Byrd, B.F., 3rd; Dokainish, H.; Edvardsen, T.; Flachskampf, F.A.; Gillebert, T.C.; Klein, A.L.; Lancellotti, P.; et al. Recommendations for the Evaluation of Left Ventricular Diastolic Function by Echocardiography: An Update from the American Society of Echocardiography and the European Association of Cardiovascular Imaging. J. Am. Soc. Echocardiogr. 2016, 29, 277-314. [CrossRef]

43. Prineas, J.R.; Crow, S.R.; Zhang, Z.-M. The Minnesota Code Manual of Electrocardiographic Findings: Standards and Procedures for Measurement and Classification, 2nd ed.; Springer: New York, NY, USA, 2010. [CrossRef]

44. Hoet, P.; Jacquerye, C.; Deumer, G.; Lison, D.; Haufroid, V. Reference values and upper reference limits for 26 trace elements in the urine of adults living in Belgium. Clin. Chem. Lab. Med. 2013, 51, 839-849. [CrossRef]

45. Salehifar, E.; Shokrzadeh, M.; Ghaemian, A.; Aliakbari, S.; Saeedi Saravi, S.S. The study of Cu and Zn serum levels in idiopathic dilated cardiomyopathy (IDCMP) patients and its comparison with healthy volunteers. Biol. Trace Elem. Res. 2008, 125, 97-108. [CrossRef]

46. Shokrzadeh, M.; Ghaemian, A.; Salehifar, E.; Aliakbari, S.; Saravi, S.S.S.; Ebrahimi, P. Serum Zinc and Copper Levels in Ischemic Cardiomyopathy. Biol. Trace Elem. Res. 2009, 127, 116-123. [CrossRef] [PubMed]

47. Nisse, C.; Tagne-Fotso, R.; Howsam, M.; Richeval, C.; Labat, L.; Leroyer, A. Blood and urinary levels of metals and metalloids in the general adult population of Northern France: The IMEPOGE study, 2008-2010. Int. J. Hyg. Environ. Health 2017, 220, 341-363. [CrossRef]

48. Wang, X.; Mukherjee, B.; Park, S.K. Associations of cumulative exposure to heavy metal mixtures with obesity and its comorbidities among U.S. adults in NHANES 2003-2014. Environ. Int. 2018, 121, 683-694. [CrossRef]

49. Rehman, K.; Fatima, F.; Waheed, I.; Akash, M.S.H. Prevalence of exposure of heavy metals and their impact on health consequences. J. Cell. Biochem. 2018, 119, 157-184. [CrossRef]

50. Nunes, J.A.; Batista, B.L.; Rodrigues, J.L.; Caldas, N.M.; Neto, J.A.; Barbosa, F., Jr. A simple method based on ICP-MS for estimation of background levels of arsenic, cadmium, copper, manganese, nickel, lead, and selenium in blood of the Brazilian population. J. Toxicol. Environ. Health Part A 2010, 73, 878-887. [CrossRef] [PubMed]

51. Waseem, A.; Arshad, J. A review of Human Biomonitoring studies of trace elements in Pakistan. Chemosphere 2016, 163, 153-176. [CrossRef] [PubMed]

52. Ding, C.; Pan, Y.; Zhang, A.; Wu, B.; Huang, H.; Zhu, C.; Liu, D.; Zhu, B.; Xu, G.; Shao, H.; et al. Study of distribution and influencing factors of arsenic in whole blood and urine among population in 8 provinces in China. Zhonghua Yu Fang Yi Xue Za Zhi [Chin. J. Prev. Med.] 2014, 48, 97-101. [PubMed] 
53. Das, A.K.; Sahu, R.; Dua, T.K.; Bag, S.; Gangopadhyay, M.; Sinha, M.K.; Dewanjee, S. Arsenic-induced myocardial injury: Protective role of Corchorus olitorius leaves. Food Chem. Toxicol. Int. J. Publ. Br. Ind. Biol. Res. Assoc. 2010, 48, 1210-1217. [CrossRef]

54. Manna, P.; Sinha, M.; Sil, P.C. Arsenic-induced oxidative myocardial injury: Protective role of arjunolic acid. Arch. Toxicol. 2008, 82, 137-149. [CrossRef] [PubMed]

55. Alamolhodaei, N.S.; Shirani, K.; Karimi, G. Arsenic cardiotoxicity: An overview. Environ. Toxicol. Pharmacol. 2015, 40, 1005-1014. [CrossRef] [PubMed]

56. Smeester, L.; Rager, J.E.; Bailey, K.A.; Guan, X.; Smith, N.; Garcia-Vargas, G.; Del Razo, L.M.; Drobna, Z.; Kelkar, H.; Styblo, M.; et al. Epigenetic changes in individuals with arsenicosis. Chem. Res. Toxicol. 2011, 24, 165-167. [CrossRef]

57. Mo, J.; Xia, Y.; Wade, T.J.; DeMarini, D.M.; Davidson, M.; Mumford, J. Altered gene expression by low-dose arsenic exposure in humans and cultured cardiomyocytes: Assessment by real-time PCR arrays. Int. J. Environ. Res. Public Health 2011, 8, 2090-2108. [CrossRef] [PubMed]

58. Domingo-Relloso, A.; Grau-Perez, M.; Briongos-Figuero, L.; Gomez-Ariza, J.L.; Garcia-Barrera, T.; Duenas-Laita, A.; Bobb, J.F.; Chaves, F.J.; Kioumourtzoglou, M.A.; Navas-Acien, A.; et al. The association of urine metals and metal mixtures with cardiovascular incidence in an adult population from Spain: The Hortega Follow-Up Study. Int. J. Epidemiol. 2019, 48, 1839-1849. [CrossRef]

59. Oster, O. Trace element concentrations $(\mathrm{Cu}, \mathrm{Zn}, \mathrm{Fe})$ in sera from patients with dilated cardiomyopathy. Clin. Chim. Acta Int. J. Clin. Chem. 1993, 214, 209-218. [CrossRef]

60. Topuzoglu, G.; Erbay, A.R.; Karul, A.B.; Yensel, N. Concentrations of copper, zinc, and magnesium in sera from patients with idiopathic dilated cardiomyopathy. Biol. Trace Elem. Res. 2003, 95, 11-17. [CrossRef]

61. Kosar, F.; Sahin, I.; Taskapan, C.; Kucukbay, Z.; Gullu, H.; Taskapan, H.; Cehreli, S. Trace element status (Se, Zn, Cu) in heart failure. Anadolu Kardiyol. Derg. Akd Anatol. J. Cardiol. 2006, 6, 216-220.

62. Cenac, A.; Simonoff, M.; Djibo, A. Nutritional status and plasma trace elements in peripartum cardiomyopathy. A comparative study in Niger. J. Cardiovasc. Risk 1996, 3, 483-487. [CrossRef]

63. Chou, H.T.; Yang, H.L.; Tsou, S.S.; Ho, R.K.; Pai, P.Y.; Hsu, H.B. Status of trace elements in patients with idiopathic dilated cardiomyopathy in central Taiwan. Zhonghua Yi Xue Za Zhi Chin. Med. J. Free China Ed. 1998, 61, 193-198.

64. Malek, F.; Dvorak, J.; Jiresova, E.; Spacek, R. Difference of baseline serum copper levels between groups of patients with different one year mortality and morbidity and chronic heart failure. Cent. Eur. J. Public Health 2003, 11, 198-201.

65. Gaetke, L.M.; Chow, C.K. Copper toxicity, oxidative stress, and antioxidant nutrients. Toxicology 2003, 189, 147-163. [CrossRef]

66. Gaetke, L.M.; Chow-Johnson, H.S.; Chow, C.K. Copper: Toxicological relevance and mechanisms. Arch. Toxicol. 2014, 88, 1929-1938. [CrossRef] [PubMed]

67. Fukai, T.; Ushio-Fukai, M.; Kaplan, J.H. Copper transporters and copper chaperones: Roles in cardiovascular physiology and disease. Am. J. Physiol. Cell Physiol. 2018, 315, C186-C201. [CrossRef]

68. Grandis, D.J.; Nah, G.; Whitman, I.R.; Vittinghoff, E.; Dewland, T.A.; Olgin, J.E.; Marcus, G.M. Wilson's Disease and Cardiac Myopathy. Am. J. Cardiol. 2017, 120, 2056-2060. [CrossRef] [PubMed]

69. WHO. Trace Elements in Human Nutrition and Health. 1996. Available online: https://apps.who.int/iris/handle/10665/37931 (accessed on 17 February 2018).

70. Canada, H. Report on Human Biomonitoring of Environmental Chemicals in Canada. Results of the Canadian Health Measures Survey Cycle 1 (2007-2009); Health Canada: Ottawa, ON, Canada, 2010.

71. Yu, X.; Huang, L.; Zhao, J.; Wang, Z.; Yao, W.; Wu, X.; Huang, J.; Bian, B. The Relationship between Serum Zinc Level and Heart Failure: A Meta-Analysis. Biomed. Res. Int. 2018, 2018, 2739014-2739019. [CrossRef]

72. Weber, K.T.; Weglicki, W.B.; Simpson, R.U. Macro- and micronutrient dyshomeostasis in the adverse structural remodelling of myocardium. Cardiovasc. Res. 2009, 81, 500-508. [CrossRef] [PubMed]

73. Choi, S.; Liu, X.; Pan, Z. Zinc deficiency and cellular oxidative stress: Prognostic implications in cardiovascular diseases. Acta Pharmacol. Sin. 2018, 39, 1120-1132. [CrossRef] [PubMed]

74. Thomas, M.; Vidal, A.; Bhattacharya, S.K.; Ahokas, R.A.; Sun, Y.; Gerling, I.C.; Weber, K.T. Zinc dyshomeostasis in rats with aldosteronism. Response to spironolactone. Am. J. Physiol. Heart Circ. Physiol. 2007, 293, H2361-H2366. [CrossRef] [PubMed]

75. Ripa, S.; Ripa, R.; Giustiniani, S. Are failured cardiomyopathies a zinc-deficit related disease? A study on $\mathrm{Zn}$ and Cu in patients with chronic failured dilated and hypertrophic cardiomyopathies. Minerva Med. 1998, 89, 397-403. [PubMed]

76. Gandhi, M.S.; Deshmukh, P.A.; Kamalov, G.; Zhao, T.; Zhao, W.; Whaley, J.T.; Tichy, J.R.; Bhattacharya, S.K.; Ahokas, R.A.; Sun, Y.; et al. Causes and consequences of zinc dyshomeostasis in rats with chronic aldosteronism. J. Cardiovasc. Pharmacol. 2008, 52, 245-252. [CrossRef]

77. Brzoska, M.M.; Moniuszko-Jakoniuk, J. Interactions between cadmium and zinc in the organism. Food Chem. Toxicol. Int. J. Publ. Br. Ind. Biol. Res. Assoc. 2001, 39, 967-980. [CrossRef]

78. Prasad, A.S.; Bao, B. Molecular Mechanisms of Zinc as a Pro-Antioxidant Mediator: Clinical Therapeutic Implications. Antioxidants 2019, 8, 164. [CrossRef]

79. Soudani, N.; Troudi, A.; Bouaziz, H.; Ben Amara, I.; Boudawara, T.; Zeghal, N. Cardioprotective effects of selenium on chromium (VI)-induced toxicity in female rats. Ecotoxicol. Environ. Saf. 2011, 74, 513-520. [CrossRef] [PubMed] 
80. Peters, J.L.; Perlstein, T.S.; Perry, M.J.; McNeely, E.; Weuve, J. Cadmium exposure in association with history of stroke and heart failure. Environ. Res. 2010, 110, 199-206. [CrossRef] [PubMed]

81. Smetana, R.H.; Glogar, D.H. Role of cadmium and magnesium in pathogenesis of idiopathic dilated cardiomyopathy. Am. J. Cardiol. 1986, 58, 364-366. [CrossRef]

82. Sundar, S.; Chakravarty, J. Antimony toxicity. Int. J. Environ. Res. Public Health 2010, 7, 4267-4277. [CrossRef]

83. Alvarez, M.; Malécot, C.O.; Gannier, F.; Lignon, J.M. Antimony-induced cardiomyopathy in guinea-pig and protection by L-carnitine. Br. J. Pharm. 2005, 144, 17-27. [CrossRef]

84. Cvjetko, P.; Cvjetko, I.; Pavlica, M. Thallium toxicity in humans. Arh. Za Hig. Rada I Toksikol. 2010, 61, 111-119. [CrossRef]

85. Osorio-Rico, L.; Santamaria, A.; Galvan-Arzate, S. Thallium Toxicity: General Issues, Neurological Symptoms, and Neurotoxic Mechanisms. Adv. Neurobiol. 2017, 18, 345-353. [CrossRef] [PubMed]

86. Hoffman, R.S. Thallium toxicity and the role of Prussian blue in therapy. Toxicol. Rev. 2003, 22, 29-40. [CrossRef] [PubMed]

87. Lin, G.; Yuan, L.; Bai, L.; Liu, Y.; Wang, Y.; Qiu, Z. Successful treatment of a patient with severe thallium poisoning in a coma using Prussian blue and plasma exchange: A case report. Medicine 2019, 98, e14629. [CrossRef]

88. Xiao, T.; Yang, F.; Li, S.; Zheng, B.; Ning, Z. Thallium pollution in China: A geo-environmental perspective. Sci. Total Environ. 2012, 421-422, 51-58. [CrossRef] [PubMed]

89. Keith, S.; Faroon, O.; Roney, N.; Scinicariello, F.; Wilbur, S.; Ingerman, L.; Llados, F.; Plewak, D.; Wohlers, D.; Diamond, G Toxicological Profile for Uranium. In Agency for Toxic Substances and Disease Registry (ATSDR) Toxicological Profiles; Agency for Toxic Substances and Disease Registry (US): Atlanta, GA, USA, 2013.

90. Bjorklund, G.; Christophersen, O.A.; Chirumbolo, S.; Selinus, O.; Aaseth, J. Recent aspects of uranium toxicology in medical geology. Environ. Res. 2017, 156, 526-533. [CrossRef]

91. Pavlakis, N.; Pollock, C.A.; McLean, G.; Bartrop, R. Deliberate overdose of uranium: Toxicity and treatment. Nephron 1996, 72, 313-317. [CrossRef] [PubMed] 